\title{
Lens galaxies in the Illustris simulation: power- law models and the bias of the Hubble constant from time delays
}

\section{Citation}

Xu, Dandan, Dominique Sluse, Peter Schneider, Volker Springel, Mark Vogelsberger, Dylan Nelson, and Lars Hernquist. 2015. "Lens Galaxies in the Illustris Simulation: Power-Law Models and the Bias of the Hubble Constant from Time Delays." Monthly Notices of the Royal Astronomical Society 456 (1): 739-55. https://doi.org/10.1093/mnras/stv2708.

\section{Permanent link}

http://nrs.harvard.edu/urn-3:HUL.InstRepos:41381763

\section{Terms of Use}

This article was downloaded from Harvard University's DASH repository, and is made available under the terms and conditions applicable to Open Access Policy Articles, as set forth at http:// nrs.harvard.edu/urn-3:HUL.InstRepos:dash.current.terms-of-use\#OAP

\section{Share Your Story}

The Harvard community has made this article openly available.

Please share how this access benefits you. Submit a story.

\section{Accessibility}




\title{
Lens galaxies in the Illustris simulation: power-law models and the bias of the Hubble constant from time-delays
}

\author{
Dandan $\mathrm{Xu}^{1 \star}$, Dominique Sluse ${ }^{2}$, Peter Schneider ${ }^{3}$, Volker Springel ${ }^{1,4}$ \\ Mark Vogelsberger ${ }^{5}$, Dylan Nelson ${ }^{6}$, Lars Hernquist ${ }^{6}$ \\ 1 Heidelberg Institute for Theoretical Studies, Schloss-Wolfsbrunnenweg 35, 69118 Heidelberg, Germany \\ 2 Institut d'Astrophysique et de Géophysique, Université de Liège, Allée du 6 Août 17, B5c, 4000 Liège, Belgium \\ 3 Argelander-Institut für Astronomie, Universität Bonn, Auf dem Hügel 71, 53121 Bonn, Germany \\ 4 Zentrum für Astronomie der Universität Heidelberg, Astronomisches Recheninstitut, Mönchhofstr. 12-14, 69120 Heidelberg, Germany \\ 5 Department of Physics, Massachusetts Institute of Technology, 77 Massachusetts Avenue, Cambridge, United States \\ ${ }^{6}$ Harvard Astronomy Department, 60 Garden Street MS 46, Cambridge, MA 02138, United States
}

Accepted ...... Received ......; in original form......

\begin{abstract}
A power-law density model, i.e., $\rho(r) \propto r^{-\gamma^{\prime}}$ has been commonly employed in strong gravitational lensing studies, including the so-called time-delay technique used to infer the Hubble constant $H_{0}$. However, since the radial scale at which strong lensing features are formed corresponds to the transition from the dominance of baryonic matter to dark matter, there is no known reason why galaxies should follow a power law in density. The assumption of a power law artificially breaks the mass-sheet degeneracy, a well-known invariance transformation in gravitational lensing which affects the product of Hubble constant and time delay and can therefore cause a bias in the determination of $H_{0}$ from the time-delay technique. In this paper, we use the Illustris hydrodynamical simulations to estimate the amplitude of this bias, and to understand how it is related to observational properties of galaxies. Investigating a large sample of Illustris galaxies that have velocity dispersion $\sigma_{\mathrm{SIE}} \geqslant 160 \mathrm{~km} \mathrm{~s}^{-1}$ at redshifts below $z=1$, we find that the bias on $H_{0}$ introduced by the power-law assumption can reach $20 \%-50 \%$, with a scatter of $10 \%-30 \%$ (rms). However, we find that by selecting galaxies with an inferred power-law model slope close to isothermal, it is possible to reduce the bias on $H_{0}$ to $\lesssim 5 \%$, and the scatter to $\lesssim 10 \%$. This could potentially be used to form less biased statistical samples for $H_{0}$ measurements in the upcoming large survey era.
\end{abstract}

Key words: gravitational lensing: strong - galaxies: haloes - galaxies: structure cosmology: theory - dark matter.

\section{INTRODUCTION}

Strong gravitational lensing is a major tool of modern extragalactic astrophysics. Ever since its first discovery (Walsh et al. 1979), it has been used as a natural telescope to magnify the distant Universe, a scale to weigh galaxies, and a ladder to measure the Hubble constant $H_{0}$ - the expansion rate of the Universe (Refsdal 1964). With the current and upcoming space missions like GAIA and Euclid, and ground-based facilities such as the Large Synoptic Survey Telescope (LSST) and the Square Kilometre Array, strong lensing stud-

^ E-mail: Dandan.Xu@h-its.org ies will experience an unprecedented opportunity to exploit several thousands of lensing galaxies to be discovered (e.g., Coe \& Moustakas 2009; Oguri \& Marshall 2010; Finet et al. 2012; LSST Dark Energy Science Collaboration 2012), while only hundreds are currently known. This transition into the big data era demands a good understanding of and control over systematic errors that are introduced by lens modelling techniques, which will affect scrutinized quantities such as the lens density slopes and their evolution with time, and derived cosmological parameters such as $H_{0}$.

The observational properties of galaxy-scale strong lens systems (i.e. positions and flux ratios of unresolved images, brightness distribution of lensed extended components, and 
time-delays) depend mainly on the mass distribution inside and near the Einstein radius of the lens, which corresponds in general to a few half-light radii. Within this radius, both dark and baryonic matter are believed to coexist in roughly similar amounts. However, because of the interplay between cooling and heating mechanisms, the unknown efficiency of star formation, and a wide variety of feedback processes, a large scatter in the galaxy total mass profiles is naively expected. Observationally, results from galaxy dynamics combined with strong lensing measurements, but also X-ray emission of elliptical galaxies, suggest a limited diversity of profiles and indicate that the inner profile is almost isothermal (e.g., Rusin et al. 2003; Humphrev et al. 2006; Gavazzi et al. 2007; Koopmans et al. 2009; Auger et al. 2010). This surprising result, also known as the "bulge-halo conspiracy", sets important constraints on the formation history of galaxies (e.g., Koopmans et al. 2006; Sonnenfeld et al. 2013; Dutton \& Treu 2014). In addition, it has an important impact on numerous phenomenological studies of galaxies, as it motivates simple power-law models i.e., $\rho(r) \propto r^{-\gamma^{\prime}}$ of the volume mass density profile, with a corresponding convergence profile $\kappa(\theta) \propto \theta^{1-\gamma^{\prime}}$ [i.e. the surface density distribution $\Sigma(\theta)$ normalized by the lensing critical density

$$
\Sigma_{\mathrm{cr}}=\left(\frac{c^{2}}{4 \pi G}\right)\left(\frac{D_{\mathrm{s}}}{D_{\mathrm{ds}} D_{\mathrm{d}}}\right),
$$

where $D_{\mathrm{d}}, D_{\mathrm{s}}$ and $D_{\mathrm{ds}}$ are the angular diameter distances to the lens, to the source, and from the lens to the source, respectively]. This model is commonly used in studies of galaxy-scale strong lens systems, and often isothermality, i.e., $\gamma^{\prime}=2$, is assumed.

Despite observational support, the power-law density profile is no more than a convenient "working model" for strong lensing studies. This family of density profiles as found by fitting strong lensing measurements does not uniquely/necessarily represent the mass distribution of the inner regions of galaxies due to the so-called mass-sheet degeneracy (MSD; Falco et al. 1985). Any given convergence field $\kappa(\boldsymbol{\theta})$ can be transformed into a new convergence profile $\kappa_{\lambda}(\boldsymbol{\theta})$ via

$$
\kappa_{\lambda}(\boldsymbol{\theta})=\lambda \kappa(\boldsymbol{\theta})+(1-\lambda) .
$$

Together with an isotropic rescaling of the source plane $\beta \rightarrow \lambda \beta$, the transformation (1) maintains the invariance of all observable quantities, such as image positions and flux ratios, except the time delays. This transformation is referred to as the "mass-sheet transformation" (MST). The only observable quantity modified under the MST is the product of the Hubble constant $H_{0}$ and the time delay $\Delta t$, which is transformed such that $H_{0} \Delta t \rightarrow \lambda H_{0} \Delta t$. Because of the invariance of observables, information from gravitational lens properties cannot distinguish between any of the members of the family of mass profiles $\kappa_{\lambda}(\boldsymbol{\theta})$. However, if a particular parametrized mass model is chosen, such as a (local or global) power-law profile, the MST is artificially broken by yielding that member of the family $\kappa_{\lambda}$ which is closest to the chosen mass profile parametrization. This artificial breaking of the MSD can lead to a systematic bias on the determination of $H_{0}$, for example, if the logarithmic density profile near the Einstein radius is systematically curved upwards or downwards relative to a power-law profile, then the values of the transformation parameter $\lambda$ will be systematically above or below unity (Schneider \& Sluse 2013).

The range of values of $\lambda$ expected from a population of lensing galaxies is currently unknown. Schneider \& Sluse (2013) have presented an example of a realistic galaxy model, composed as the sum of a baryonic and a dark matter profile, which could be transformed into an approximate power-law density profile through Eq. (1) with $\lambda \sim 1.2$, leading to a systematic error of about $20 \%$ on $H_{0}$. Van de Ven et al. (2009) have shown that a large variety of observationally motivated composite models of galaxies systematically produce profiles that are slightly more convex/concave than isothermal in regions probed by lensing. Here, we study the expected distribution of surface density profiles of galaxies drawn from a cosmological hydrodynamic simulation - the Illustris Project Vogelsberger et al. 2014, see also Vogelsberger et al. 2014; Genel et al. 2014; Nelson et al. 2015). By selecting galaxies that most resemble the observed lensing galaxies in term of mass/velocity dispersion, we test the validity of the power-law assumption in the central regions of galaxies, and evaluate the expected distribution of the multiplicative bias $\lambda$ on $H_{0}$ for this sample. We emphasize that in this analysis we assume that no other external constraint than the lensing information is available to break the MSD. Whereas stellar dynamical information from spectroscopy can yield additional constraints on the lensing mass distribution and thus helps to limit the allowed range of $\lambda$, the accuracy of this method is limited, e.g., by spectroscopic resolution and the unknown distribution of stellar orbits.

The outline of the paper is as follows. In Sect. 2, we describe the methodology to measure the slope and curvature of the surface density profiles of the simulated galaxies, and how we derive the $\lambda$ parameter which transforms the intrinsic distribution into an approximate power law. In Sect. 3 , we give a brief description of the lensing galaxy samples that we have extracted from the Illustris simulation; general properties of the simulated galaxy sample are presented. As the (surface) density distributions are of particular interest, we dedicate Sect. 4 to a detailed discussion about the diversity of the central density profiles in regions probed by strong lensing. In Sect. 5, we present distributions of $\lambda$ that result from the use of power-law density profiles to model various samples of lensing galaxies. Such distributions also indicate by how much the derived value of $H_{0}$ would be systematically biased from the true value by employing the power-law assumption. A final discussion and conclusions are given in Sect. 6 .

\section{METHODOLOGY}

In this section we explain the quantities that we use to characterize the density profiles of the simulated galaxies drawn from Illustris. Since the MST only affects the radial density profile, but leaves the shape of the isodensity contours invariant, we are only concerned with the azimuthally-averaged, i.e., radial density profile $\kappa(\theta)$ of a lensing galaxy.

In order to quantify how well power-law models can describe the surface density profile of a lensing galaxy, we have first calculated a mean local slope $s$ and the corresponding curvature $\xi$ following the treatment of Schneider \& Sluse 
(2013). Assuming that the strong lensing features (multiple compact images, images of extended source components) are located between an inner and an outer radius of $\theta_{1}=x_{1} \theta_{\mathrm{E}}$ and $\theta_{2}=x_{2} \theta_{\mathrm{E}}$, respectively, where $\theta_{\mathrm{E}}$ is the Einstein radius of the lens, a mean logarithmic slope $s$ for the convergence distribution $\kappa(\theta)$ between $\theta_{1}$ and $\theta_{2}$ can then be calculated as 1

$$
s \equiv \frac{\ln \left(\kappa_{2} / \kappa_{1}\right)}{\ln \left(\theta_{1} / \theta_{2}\right)}
$$

where $\kappa_{1} \equiv \kappa\left(\theta_{1}\right)$ and $\kappa_{2} \equiv \kappa\left(\theta_{2}\right)$ are the convergence at the inner and outer image positions. $s=1$ corresponds to the projected slope expected for an isothermal distribution; $s>1(s<1)$ indicates the profile steeper (shallower) than isothermal. Unless mentioned otherwise, we set $x_{1}=0.5$ and $x_{2}=1.5$ in this paper, which are characteristic values for observed lensed systems.

We define the curvature parameter $\xi$ as

$$
\xi \equiv \frac{\kappa\left(\sqrt{\theta_{1} \theta_{2}}\right)}{\sqrt{\kappa_{1} \kappa_{2}}},
$$

which indicates the closeness of the profile $\kappa(\theta)$ between $\theta_{1}$ and $\theta_{2}$ to a power-law distribution: an exact power-law profile will have $\xi=1$; while $\xi>1(\xi<1)$ means that the projected density profile is concave-upward (convex-downward), which lies above (below) the power-law interpolation between $\theta_{1}$ and $\theta_{2}$.

The MST (1) transforms the original $\kappa(\theta)$ into a new distribution $\kappa_{\lambda}(\theta)$, with slope

$$
s_{\lambda}=\frac{\ln \left(\kappa_{\lambda}\left(\theta_{2}\right) / \kappa_{\lambda}\left(\theta_{1}\right)\right)}{\ln \left(\theta_{1} / \theta_{2}\right)}
$$

and curvature

$$
\xi_{\lambda}=\frac{\kappa_{\lambda}\left(\sqrt{\theta_{1} \theta_{2}}\right)}{\sqrt{\kappa_{\lambda}\left(\theta_{1}\right) \kappa_{\lambda}\left(\theta_{2}\right)}},
$$

leaving image astrometry and flux ratios unchanged. Here and below, we specifically denote $\lambda$ as the value of the transformation parameter that transforms $\kappa(\theta)$ to a new distribution $\kappa_{\lambda}(\theta)$, which satisfies the criterion that $\xi_{\lambda}=1$; this condition yields

$$
\lambda=\frac{\kappa_{2}+\kappa_{1}-2 \xi \sqrt{\kappa_{2} \kappa_{1}}}{\kappa_{2}+\kappa_{1}-2 \xi \sqrt{\kappa_{2} \kappa_{1}}+\left(\xi^{2}-1\right) \kappa_{2} \kappa_{1}} .
$$

Let $r \equiv \theta_{1} / \theta_{2}<1$, so that $\kappa_{2} / \kappa_{1}=r^{s}$ (from Eq. 2). Then by dividing the numerator and denominator of Eq. (44) by $\kappa_{1}$, we can re-write $\lambda$ as a function of $r, s, \xi$ and $\kappa_{2}$ :

$$
\lambda=\frac{1+r^{s}-2 \xi r^{s / 2}}{1+r^{s}-2 \xi r^{s / 2}+\left(\xi^{2}-1\right) \kappa_{2}} .
$$

Note that in the case where $\lambda=1, \xi=\xi_{\lambda}=1$. Here and in the following, we use $\xi_{\lambda}=1$ as our powerlaw criterion: that an MST with $\lambda$ given by Eq. (5) transforms $\kappa(\theta)$ into an approximate power law $\kappa_{\lambda}(\theta)$ between $\theta_{1}$ and $\theta_{2}$ is in such a sense that the three points $\left(\ln \theta_{1}, \quad \ln \kappa_{\lambda}\left(\theta_{1}\right)\right), \quad\left(\ln \theta_{2}, \quad \ln \kappa_{\lambda}\left(\theta_{2}\right)\right)$ and their mid-point $\left(\ln \sqrt{\theta_{1} \theta_{2}}, \ln \kappa_{\lambda}\left(\sqrt{\theta_{1} \theta_{2}}\right)\right)$ lie on a straight line in the logarithmic $\kappa(\theta)$ plot.

A meaningful MST requires that (i) the transformed

1 The quantity that we have denoted here as $s$ is actually the same as $\bar{s}$ of Schneider \& Sluse (2013). density profile $\kappa_{\lambda}(\theta)$ remains monotonically decreasing, which yields $\lambda>0$; and (ii) $\kappa_{\lambda}(\theta)$ is non-negative over the range considered, i.e., $\kappa_{\lambda}\left(\theta_{2}\right)>0$, which yields $\lambda<$ $1 /\left(1-\kappa_{2}\right)$. Note that typically $85 \%$ of the galaxies in our lens samples satisfy condition (i) and all satisfy condition (ii). See Table 1 for the exact fractions.

The slope $s_{\lambda}$ of the transformed profile, which by construction is an approximate power law between $\theta_{1}$ and $\theta_{2}$ (i.e., $\xi_{\lambda}=1$ ), can explicitly be related to the properties of the original profile. Indeed, by definition it obeys the relation

$$
r^{s_{\lambda}}=\frac{\kappa_{\lambda}\left(\theta_{2}\right)}{\kappa_{\lambda}\left(\theta_{1}\right)}=\frac{\lambda \kappa_{2}+(1-\lambda)}{\lambda \kappa_{1}+(1-\lambda)}=\frac{\kappa_{2}+(1-\lambda) / \lambda}{\kappa_{1}+(1-\lambda) / \lambda} .
$$

From Eq. (4) we obtain

$$
\frac{1-\lambda}{\lambda}=\frac{\left(\xi^{2}-1\right) \kappa_{1} \kappa_{2}}{\kappa_{1}+\kappa_{2}-2 \xi \sqrt{\kappa_{1} \kappa_{2}}} .
$$

Combining Eq. (7) with Eq. (6) then yields

$$
r^{s_{\lambda}}=\frac{\kappa_{2}^{2}-2 \xi \kappa_{2} \sqrt{\kappa_{1} \kappa_{2}}+\xi^{2} \kappa_{1} \kappa_{2}}{\kappa_{1}^{2}-2 \xi \kappa_{1} \sqrt{\kappa_{1} \kappa_{2}}+\xi^{2} \kappa_{1} \kappa_{2}} .
$$

By dividing the numerator and denominator of Eq. (8) by $\kappa_{1} \kappa_{2}$ we obtain

$$
\begin{aligned}
r^{s_{\lambda}} & =\frac{r^{s}-2 \xi r^{s / 2}+\xi^{2}}{r^{-s}-2 \xi r^{-s / 2}+\xi^{2}} \\
& =r^{s}\left(\frac{\xi-r^{s / 2}}{1-\xi r^{s / 2}}\right)^{2} \\
& =r^{s}\left(1+\frac{\left(r^{-s}-1\right)\left(\xi^{2}-1\right)}{\left(r^{-s / 2}-\xi\right)^{2}}\right) .
\end{aligned}
$$

Hence, the power-law slope after MST is

$$
s_{\lambda}=s+\ln \left(1+\frac{\left(r^{-s}-1\right)\left(\xi^{2}-1\right)}{\left(r^{-s / 2}-\xi\right)^{2}}\right) / \ln r .
$$

Thus, $s_{\lambda}$ is a function solely of the original slope $s$ and the curvature parameter $\xi$. If $\xi>1$, then (since $r^{-s}>1$ and $\ln r<0) s_{\lambda}<s$, i.e., in this case the transformed profile is flatter than the original. This case also corresponds to $\lambda<1$, i.e., the mass sheet added has positive convergence, leading to a flatter profile after MST.

It is worth noting that a different radial range $\left[\theta_{1}, \theta_{2}\right]$ would yield different $\kappa_{\lambda}$ and $\lambda$. This is simply because they depend on the slope $s$ and curvature $\xi$ which are defined in terms of the angular range where strong lensing measurements are available. As stated before, we focus on the range $\theta_{1}=0.5 \theta_{\mathrm{E}}$ and $\theta_{2}=1.5 \theta_{\mathrm{E}}$. Results for a different angular range (with $\theta_{1}=0.8 \theta_{\mathrm{E}}$ and $\theta_{2}=1.2 \theta_{\mathrm{E}}$ ) are also presented in Appendix A As can be seen, although measurements in these two cases are not exactly the same on a one-to-one basis, the statistical distributions of $\lambda$ are independent of the choice of the $\theta_{i}(i=1,2)$.

Equations (1) to (10) relate to the local convergence profile $\kappa(\theta)$. They were derived and discussed in the framework of the assumption that the convergence profile follows approximately a power law in the range $\theta_{1} \leqslant \theta \leqslant \theta_{2}$. Alternatively, we can also define analogous quantities under the assumption that the radial dependence of the deflection angle behaves like an approximate power law, which is equivalent to a power law in the mean convergence within 
$\theta$, i.e.,

$$
\bar{\kappa}(\theta)=\frac{2}{\theta^{2}} \int_{0}^{\theta} \mathrm{d} \theta^{\prime} \theta^{\prime} \kappa\left(\theta^{\prime}\right) .
$$

Hence, we can consider an MST that results in a transformed cumulative distribution $\bar{\kappa}_{\lambda}$ to satisfy our power-law criterion so that the profile of $\bar{\kappa}_{\lambda}$ is a good approximation of a power law between $\theta_{1}$ and $\theta_{2}$. The MST for $\bar{\kappa}$ is the same as that for $\kappa$, i.e.,

$$
\bar{\kappa}_{\lambda}=\lambda \bar{\kappa}+(1-\lambda) .
$$

Note that the radii where $\kappa=\kappa_{\lambda}=1$ and where $\bar{\kappa}=\bar{\kappa}_{\lambda}=1$ remain unchanged; the latter corresponds to the Einstein radius by definition. Hereafter we denote by barred symbols all quantities that are associated with the cumulative distribution $\bar{\kappa}$ and its MST. The whole formalism from Eq. (2) to (10) also holds for $\bar{\kappa}$ after replacing $\kappa$ with $\bar{\kappa}, s$ and $\xi$ with $\bar{s}$ and $\bar{\xi}, s_{\lambda}$ and $\xi_{\lambda}$ with $\bar{s}_{\lambda}$ and $\bar{\xi}_{\lambda}$, and $\lambda$ with $\bar{\lambda}$.

Which of the two MSTs - the one that yields an approximate local power law of the convergence, i.e., $\xi_{\lambda}=1$, or that leading to an approximate power law in the deflection, i.e., $\bar{\xi}_{\lambda}=1$ - is the more relevant one depends on which assumptions are made in lens modelling. In many applications, it is assumed that the density profile follows a global power law (at least up to the radius where strong lensing features are observed), in which case the requirement $\bar{\xi}_{\lambda}=1$ applies. Alternatively, lens models can be considered in which the local profile follows a power law, but an additional mass component must be assumed on angular scales smaller than $\theta_{1}$, i.e., the presence of a supermassive black hole, or a finite core radius of the mass distribution, both of which cause deviations from a global power law. Note that the assumption of a global power law implies that the density profile between $\theta_{1}$ and $\theta_{2}$ is a local power law (with the same slope). Thus, the global power-law assumption is a stronger one than just enforcing a local power law. However, since the density profiles of real galaxies do not follow power laws, this conclusion does not hold for real lenses. We note that in general, $\lambda \neq \bar{\lambda}$, and $s_{\lambda} \neq \bar{s}_{\lambda}$.

\section{THE ILLUSTRIS LENS SAMPLES AND THEIR GENERAL PROPERTIES}

The Illustris Project is a series of cosmological hydrodynamical simulations of galaxy formation Vogelsberger et al. 2014, see also Vogelsberger et al. 2014; Genel et al. 2014; Nelson et al. 2015). The highest resolution run covers a volume of $(106.5 \mathrm{Mpc})^{3}$ and has a dark matter mass resolution of $6.26 \times 10^{6} M_{\odot}$ and an initial baryonic mass resolution of $1.26 \times 10^{6} M_{\odot}$, resolving gravitational dynamics down to a physical scale of $\epsilon=710 \mathrm{pc}$. Taking into account various baryonic processes (Vogelsberger et al. 2013, 2014), such as gas cooling, stellar evolution and feedback, chemical enrichment, supermassive black hole growth and feedback from active galactic nuclei, the Illustris simulation resolves 40000 galaxies with a variety of morphologies and reproduces many fundamental properties of observed galaxies (e.g., galaxy luminosity functions and Tully-Fisher relations).

The Illustris Project is an ideal "laboratory" for our study. On the one hand, this is because galaxy-scale strong lensing probes the projected central regions of galaxies where baryons yield a significant contribution to the total mass profile. On the other hand, the Illustris simulation provides a large and realistic sample of galaxies as needed to quantify in a statistical way the systematics associated with the assumption of power law density profiles in stronglensing studies.

\subsection{Main samples of lensing galaxies}

We take the lensing galaxies at various redshifts from the mock strong-lens catalogue of the Illustris simulation (Nelson et al. 2015, see also Xu et al., in preparation). Our selection criteria are as follows: for a given source redshift $z_{\mathrm{s}}$, the angular (and physical) Einstein radius $\theta_{\mathrm{E}}$ (and $R_{\mathrm{E}}$ ) of each galaxy is first determined as the radius within which the mean convergence $\bar{\kappa}=1$. Referring to the singular isothermal sphere model, we characterize each lens galaxy by its "velocity dispersion" $\sigma_{\mathrm{SIE}}$, which is linked to the Einstein radius via $\theta_{\mathrm{E}}=4 \pi\left(\sigma_{\mathrm{SIE}} / c\right)^{2}\left(D_{\mathrm{ds}} / D_{\mathrm{s}}\right)$. We select galaxies that have $\sigma_{\text {SIE }} \geqslant 160 \mathrm{~km} \mathrm{~s}^{-1}$. This lower limit on the velocity dispersion is motivated by the observed lens sample from the SLACS survey, where stellar velocity dispersions of lens galaxies range from $160 \mathrm{~km} \mathrm{~s}^{-1}$ to $400 \mathrm{~km} \mathrm{~s}^{-1}$ Bolton et al. 2008).

The Illustris simulation does not sample the very massive end of the mass spectrum well, i.e., galaxy clusters. There are a couple of massive systems that have total masses around $10^{14} h^{-1} M_{\odot}$. We have adopted a further selection criterion, i.e., excluding all satellite/companion galaxies in the group and galaxy environment and only selecting the "central" galaxies to form our sample. The projection effects from their companions (as well as galaxies along the line of sight) have been excluded from the calculation of the density profiles of these "central" galaxies.

Note that the surface brightness distributions of the Illustris lensing galaxies can be well fitted by Sersic profiles (Sérsic 1963). In particular for the elliptical galaxie: $2^{2}$ whose stellar masses and velocity dispersions are in the same ranges as the SLACS samples, their logarithmic density slopes are also consistent with the one constrained for the SLACS samples (e.g., Auger et al. 2010; Sonnenfeld et al. 2013). A detailed comparison of Illustris galaxies to the SLACS sample will be presented in a forthcoming paper (Xu et al., in preparation). In this paper, as we are interested in the surface density distribution, which is a projected quantity, and in order to increase the sample size, we treat the three independent projections of each selected galaxy as independent lenses. This yields more than 1000 surface density profiles for the analysis at each of the lens redshifts studied.

In the following we refer to the region where strong lensing measurements are available (i.e., the range $\left.\left[\theta_{1}, \theta_{2}\right]\right)$ as the strong lensing region. Because $\theta_{\mathrm{E}}$ is an angular quantity, the strong lensing region depends on the lens redshift $z_{\mathrm{d}}$ and the source redshift $z_{\mathrm{s}}$. In order to separate the effect of galaxy evolution from a change of $R_{\mathrm{E}}$ (such that lensing probes different regions of a same galaxy), we have formed

2 The surface brightness distribution of each galaxy in the mock catalogue has been fitted using both the de Vaucouleurs and the exponential disc profiles. A galaxy is classified as an early (late) type if the former (latter) provides a better fit. 
Table 1. A summary of sample properties (galaxies in their three independent projections with $\sigma_{\mathrm{SIE}} \geqslant 160 \mathrm{~km} \mathrm{~s}^{-1}$ are selected). The total number of galaxy projections that meet the selection criterion are given in row (3); row (4), (5) and (6) give the percentages of galaxies whose profiles can be transformed to power laws via MST (see Sect. 2); $R_{\mathrm{E}}^{\mathrm{min}}$ in row (7) is the minimal physical Einstein radius, which corresponds to the lower limit of $\sigma_{\mathrm{SIE}}=160 \mathrm{~km} \mathrm{~s}^{-1}$; in row (8), (9) and (10), the mean and medi an Einstein radius and its standard deviation $\sigma_{R_{\mathrm{E}}}$ are presented, respectively.

\begin{tabular}{|c|c|c|c|c|c|c|c|}
\hline \multirow{2}{*}{$\begin{array}{c}\text { Sample sets } \\
\text { Redshifts }\end{array}$} & \multicolumn{4}{|c|}{$z_{\mathrm{s}}=1.5$} & \multicolumn{3}{|c|}{$z_{\mathrm{d}}=0.6$} \\
\hline & $z_{\mathrm{d}}=0.2$ & $z_{\mathrm{d}}=0.4$ & $z_{\mathrm{d}}=0.6$ & $z_{\mathrm{d}}=0.8$ & $z_{\mathrm{s}}=1.0$ & $z_{\mathrm{s}}=1.5$ & $z_{\mathrm{s}}=3.0$ \\
\hline Total number of projections & 1044 & 1334 & 1433 & 1363 & 1092 & 1433 & 1673 \\
\hline Meaningful MST for $\kappa$ & $90 \%$ & $93 \%$ & $90 \%$ & $88 \%$ & $88 \%$ & $90 \%$ & $93 \%$ \\
\hline Meaningful MST for both $\kappa$ and $\bar{\kappa}$ & $76 \%$ & $81 \%$ & $77 \%$ & $73 \%$ & $72 \%$ & $77 \%$ & $80 \%$ \\
\hline$R_{\mathrm{E}}^{\min }(\mathrm{kpc})$ & 2.00 & 2.60 & 2.50 & 2.06 & 1.68 & 2.50 & 3.29 \\
\hline Mean $R_{\mathrm{E}}(\mathrm{kpc})$ & 4.16 & 5.32 & 5.14 & 4.41 & 3.71 & 5.14 & 6.47 \\
\hline
\end{tabular}

two sets of samples according to different combinations of $z_{\mathrm{s}}$ and $z_{\mathrm{d}}$

In the first set, we have selected galaxies at $z_{\mathrm{d}}=$ $[0.2, \quad 0.4,0.6,0.8]$, assuming a fixed source redshift at $z_{\mathrm{s}}=1.5$ (Table 1, left-hand columns). Note that assuming the innermost boundary of the strong lensing region to be $0.5 R_{\mathrm{E}}$, the $z_{\mathrm{d}}-z_{\mathrm{s}}$ combinations used here guarantee that even the smallest galaxies (with Einstein radius of $R_{\mathrm{E}}^{\min }$, which corresponds to the lower limit of $\sigma_{\mathrm{SIE}}=160 \mathrm{~km} \mathrm{~s}^{-1}$ ) are fully resolved at radii larger than the innermost boundary, i.e., $0.5 R_{\mathrm{E}}^{\mathrm{min}} \gtrsim \epsilon$ (where $\epsilon=710 \mathrm{pc}$ is the simulation softening length). As can be seen from Table 1 the mean Einstein radius of the galaxies, $\left\langle R_{\mathrm{E}}\right\rangle$, is approximately the same for the four $z_{\mathrm{d}}-z_{\mathrm{s}}$ combinations. We therefore probe with this sample typically the inner $5 \mathrm{kpc}$ of galaxies, such that any difference in the final statistical results is due to galaxy evolution.

In the second sample set, we fix the lens redshift at $z_{\mathrm{d}}=0.6$ and assume source redshifts at $z_{\mathrm{s}}=[1.0,1.5,3.0]$ (Table 1 right-hand columns). Again the $z_{\mathrm{d}}-z_{\mathrm{s}}$ combinations applied in this sample set also guarantee that the smallest galaxies are fully resolved at radii larger than the innermost boundary of their strong lensing regions. For fixed $z_{\mathrm{d}}$, the higher $R_{\mathrm{E}}$ correspond to the larger $z_{\mathrm{s}}$ : the mean $\left\langle R_{\mathrm{E}}\right\rangle$ for the $z_{\mathrm{s}}=3.0$ sample is nearly twice as large as that for the $z_{\mathrm{s}}=1.0$ sample. This allows us to study the dependence of our results on the radii probed by strong lensing. Note that different numbers of galaxies are obtained in spite of the same $\sigma_{\text {SIE }}$ cut, because the actual galaxy mass distributions are not truly isothermal, which thus introduces scatter in the $\sigma_{\mathrm{SIE}}$-mass relation. The scatter in mass that corresponds to $\sigma_{\mathrm{SIE}}=160 \mathrm{~km} \mathrm{~s}^{-1}$ for the $z_{\mathrm{s}}=1.0$ sample results in a minimum halo mass of $\sim 9 \times 10^{11} h^{-1} M_{\odot}$; while the scatter in the $z_{\mathrm{s}}=3.0$ sample leads to a slightly lower halo-mass limit of $\sim 7 \times 10^{11} h^{-1} M_{\odot}$. The latter sample therefore has a larger number of galaxies than the former.

\subsection{Galaxy profiles and general properties}

In the following, we present the surface density profiles of the selected galaxies and some of their general properties.

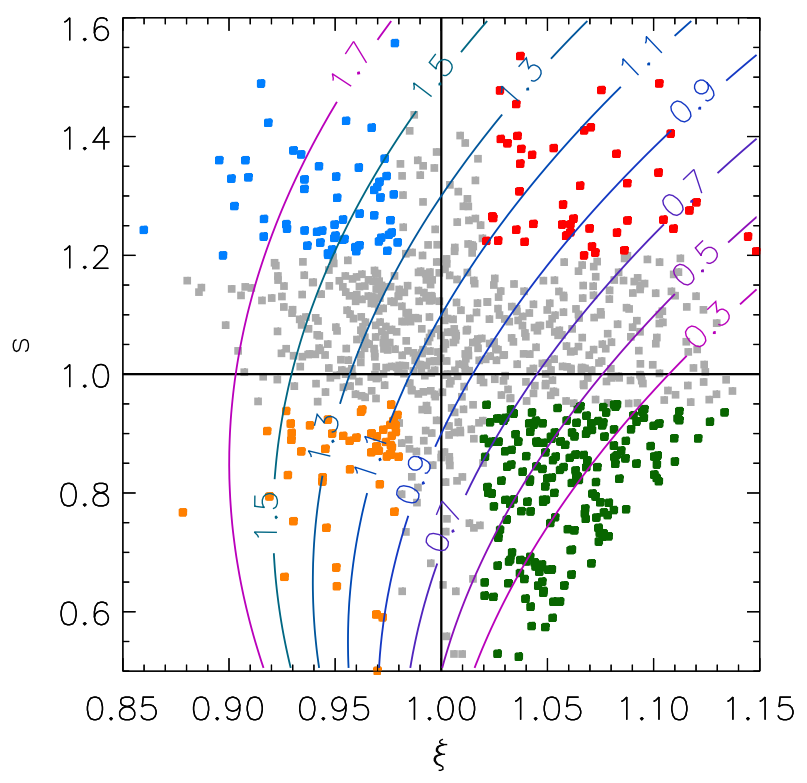

Figure 1. The $s-\xi$ distribution of the selected lensing galaxies at $z_{\mathrm{d}}=0.2$, assuming $z_{\mathrm{s}}=1.5$. The sample is divided into four subgroups according to their $s$ and $\xi$ values: subgroup I (red) is defined as galaxies with $s>1.2, \xi>1.02$; subgroup II (blue) are those with $s>1.2, \xi<0.98$; subgroup III (orange) represents galaxies with $s<0.95, \xi<0.98$; and subgroup IV (green) are those with $s<0.95, \xi>1.02$. Contours indicate where the transformed slopes $s_{\lambda}=[0.3,0.5,0.7,0.9,1.1,1.3,1.5,1.7]$.

For each galaxy, we have azimuthally averaged 3 the surface mass density distribution to obtain the radial profile of the convergence in the projected central region. To derive the profile parameters, we fit a 10 -order polynomial to the log-

3 We have also calculated the radial profile by averaging the convergence distribution in elliptical annuli, the shape of which is determined by the second moment of total projected mass within $2 \theta_{\mathrm{E}}$. We have verified that both the curvature parameter $\xi$ and the MST parameters $\lambda$ under the elliptical average are consistent with the ones derived under azimuthal averaging; the final result remains. 
arithmic radial profile within a radius range from $2 \epsilon$ to five times the half-stellar-mass radius of the galaxy, which well covers the region constrained by strong lensing. Using the polynomial fitted radial profiles, we measure the slopes and curvatures of both the local convergence $\kappa$ and the cumulative distribution $\bar{\kappa}$, which are then used to derive the MST parameters $\lambda$ and $\bar{\lambda}$ and the transformed slopes $s_{\lambda}$ and $\bar{s}_{\lambda}$, according to the formalisms derived in Sect. 2 .

Figure 1 shows the distribution of $s$ versus $\xi$ for the lens sample with $z_{\mathrm{d}}=0.2$ and $z_{\mathrm{s}}=1.5$. The overlaid contours indicate the transformed slope $s_{\lambda}$ as a function of $(s$, $\xi)$, which increases from the bottom right quadrant to the top-left quadrant in the $s-\xi$ plane. The mean slope $s$ measured between $0.5 \theta_{\mathrm{E}}$ and $1.5 \theta_{\mathrm{E}}$ typically ranges from 0.5 to 1.6 , while for the curvature $\xi$, we have $|\xi-1| \lesssim 15 \%$ for all our samples. Of course, the range of $\xi$ strongly depends on $\Delta \theta \equiv \theta_{2}-\theta_{1}:$ as $\Delta \theta \rightarrow 0, \xi \rightarrow 1$. Note that galaxies that fail the criteria " $\lambda>0$ " (and thus $s_{\lambda}>0$ ) for a meaningful MST lie at the lower-right corner of the diagram, to the right of the green points. These galaxies are not fundamentally different from the "green" galaxies in terms of their physical properties except that they have even shallower profiles with larger curvatures. Mathematically in order to transform their profiles into power laws, the additional mass sheet $(1-\lambda)$ has to be so large that the transformed powerlaw densities become lower in the centres and larger at the outskirts, and thus $s_{\lambda}$ becomes negative.

In order to understand different profile behaviours, we have further divided each lens sample into four representative subgroups according to their $s$ and $\xi$ values. As shown by the coloured symbols in Fig. 1 subgroup I as indicated by red is defined for galaxies with $s>1.2$ and $\xi>1.02$, i.e., galaxies with mean slopes steeper than isothermal and having concave-upward profiles; subgroup II as shown in blue consists of galaxies with $s>1.2$ and $\xi<0.98$, i.e., galaxies that also have mean slopes steeper than isothermal but convex-downward profiles; subgroup III as marked in orange represents galaxies with $s<0.95, \xi<0.98$, i.e., those with mean slopes flatter than isothermal and with convexdownward profiles; and subgroup IV plotted in green is composed of galaxies with $s<0.95, \xi>1.02$, i.e., those with mean slopes flatter than isothermal but having concaveupward profiles.

Since $\xi=1$ indicates approximate power-law profiles, it is used to separate subgroups. The division using $s=1$ is somewhat arbitrary; this is purely motivated by the observation that the distribution of the total density slopes of lens galaxies seems to peak at the isothermal slope (Rusin et al. 2003; Rusin \& Kochanek 2005; Koopmans et al. 2006, 2009; Gavazzi et al. 2007; Auger et al. 2010). In other words, the four subgroups are extreme cases where the density profiles are far from isothermal and perfect power law.

Apart from the profile parameters, for each galaxy in projection we have also calculated a variety of properties: (1) the total stellar mass $M_{\star}$ of the galaxy and the total mass $M_{\text {tot }}$ of the galaxy plus its host dark matter halo; (2) the effective radius $\theta_{\text {eff }}$, within which half of the galaxy luminosity is enclosed; (3) the cumulative dark matter fraction $f_{\mathrm{dm}}\left(\leqslant \theta_{\mathrm{E}}\right)$ projected within $\theta_{\mathrm{E}}$ and the local dark matter fraction $f_{\mathrm{dm}}\left(\theta_{\mathrm{E}}\right)$ projected at $\theta_{\mathrm{E}} ;(4)$ the angular radius $\theta_{f 50}$ where the projected density distribution of dark matter in-
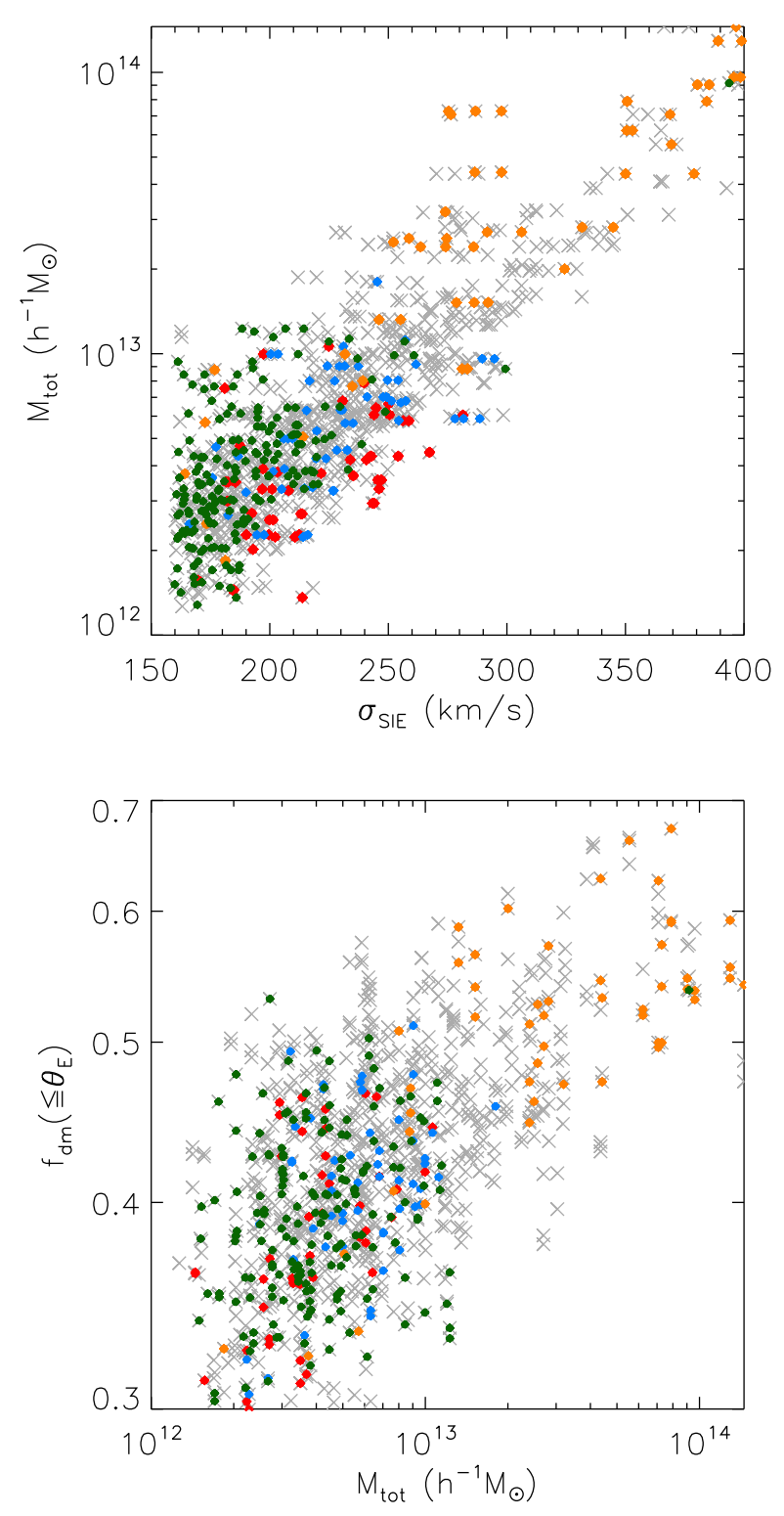

Figure 2. The distributions of $M_{\text {tot }}$ versus $\sigma_{\mathrm{SIE}}$ (top) and $f_{\mathrm{dm}}$ ( $\leqslant$ $\left.\theta_{\mathrm{E}}\right)$ versus $M_{\text {tot }}$ (bottom). The galaxy sample as well as the colour coding is the same as used in Fig. 1

tercepts that of the baryonic matter, i.e., the radius where the local dark matter fraction $f_{\mathrm{dm}}\left(\theta_{f 50}\right)=0.5$.

Fig. 2 shows the distributions of $M_{\text {tot }}$ versus $\sigma_{\text {SIE }}$ (top) and $f_{\mathrm{dm}}\left(\leqslant \theta_{\mathrm{E}}\right)$ versus $M_{\mathrm{tot}}$ (bottom) for the same galaxy sample as in Fig. 1 Different projections of the same galaxy result in multiple values of $\sigma_{\mathrm{SIE}}$ and $f_{\mathrm{dm}}\left(\leqslant \theta_{\mathrm{E}}\right)$ at the same mass, reflecting the triaxiality of the lens mass distribution. The cumulative dark matter fraction $f_{\mathrm{dm}}\left(\leqslant \theta_{\mathrm{E}}\right)$ typically ranges from $30 \%$ to $70 \%$ with more massive galaxies having higher $f_{\mathrm{dm}}\left(\leqslant \theta_{\mathrm{E}}\right)$, in good agreement with strong lensing observations (e.g., Treu \& Koopmans 2004; Koopmans et al. 2006; Jiang \& Kochanek 2007; Tortora et al. 2009; Grillo et al. 2009; Cardone et al. 2009; Napolitano et al. 2010).

It can be seen clearly that the four subgroups have 
different galaxy properties, e.g., the shallower- and convexprofiled galaxies (subgroup III) are among the most massive ones and have the largest velocity dispersions and the highest dark matter fractions. In the next section, we aim at understanding the direct reason for such a diversity in the surface density profiles measured in strong lensing regions.

\section{THE CENTRAL SURFACE DENSITY PROFILE: SLOPE AND CURVATURE}

Galaxy-scale strong lensing probes the central few kpc region of a lensing galaxy. The central (surface) density profile is of particular interest, because on such scales, the enclosed mass of dark and baryonic matter are quite similar. Various processes associated with star formation and feedback together shape the density distributions of both components and their sum.

We first illustrate in Fig. 3 the projected density profiles associated with the four galaxy subgroups (Sect. 3.2). For each subgroup we show ten typical density profiles and their dark matter fraction distributions. The projected radii are normalized to $\theta_{\mathrm{E}}$ (see Fig. B2 for profiles as a function of $\left.\theta_{\text {eff }}\right)$. In the top panels, the black curves are the total surface density distributions $\kappa(\theta)$, scaled up by a factor of 5 for clarity; the red and blue curves represent profiles of the projected dark matter $\kappa(\theta) f_{\mathrm{dm}}(\theta)$ and projected baryonic mass $\kappa(\theta)\left[1-f_{\mathrm{dm}}(\theta)\right]$, respectively; the dashed green line indicates the logarithmic slope of $s=1$ from $0.5 \theta_{\mathrm{E}}$ to $1.5 \theta_{\mathrm{E}}$. In the bottom panels, the cumulative and local dark matter fraction $f_{\mathrm{dm}}(\leqslant \theta)$ and $f_{\mathrm{dm}}(\theta)$ are given in black and green, respectively.

The first conclusion is that, as expected, the baryonic distribution is much more concentrated than the dark matter distribution. In regions probed by strong lensing, the two profiles intercept; the slope of the former is in general steeper than that of the latter. As a result, baryons and dark matter dominate different parts of the total (surface) density profile: the former in the inner regions and the latter at larger radii; the dark matter fraction therefore increases with radius.

\subsection{Individual profiles of the two ingredients}

As the total density profile is the sum of two components, we present hereafter slopes $s$ and curvatures $\xi$ of the baryonic (i.e. $s_{\mathrm{b}}$ and $\xi_{\mathrm{b}}$ ) and dark matter profile (i.e. $s_{\mathrm{dm}}$ and $\xi_{\mathrm{dm}}$ ) separately.

Fig. 4 and 5$]$ show the dependencies of the slopes and curvatures on $\theta_{f 50} / \theta_{\mathrm{E}}$ and $f_{\mathrm{dm}}\left(\theta_{\mathrm{E}}\right)$, which are two key quantities that are closely related to different profiles of subgroups. In particular, $\theta_{f 50} / \theta_{\mathrm{E}}$ describes where, with respect to $\theta_{\mathrm{E}}$, the fraction of the projected dark matter catches up and the distribution intercepts that of baryons. Different values of $\theta_{f 50} / \theta_{\mathrm{E}}$ reflect different parts of the profile that strong lensing probes: a smaller $\theta_{f 50} / \theta_{\mathrm{E}}$ means that the strong lensing region $\left(\sim \theta_{\mathrm{E}}\right)$ is at larger radii than the baryon-dark matter interception radius $\theta_{f 50}$, and thus the dark matter fraction $f_{\mathrm{dm}}\left(\theta_{\mathrm{E}}\right)$ is larger. As indicated by the colour coding, in the four highlighted subgroups, strong lensing probes different parts of the density profile, from much further-out in subgroup III (orange) to most closer-in in subgroup IV (green).

As can be seen from Fig. 4, the central density profile of the dark matter distribution is always shallower than isothermal, i.e., $s_{\mathrm{dm}}<1$; while that of the baryonic matter in most cases is considerably steeper, i.e., $s_{\mathrm{b}}>1$. From subgroup IV (green) to I (red), to II (blue) and to III (orange), as strong lensing probes increasingly larger radii of the galaxy, a significant profile steepening followed by a slope flattening develops with increasing $f_{\mathrm{dm}}\left(\theta_{\mathrm{E}}\right)$ (or decreasing $\left.\theta_{f 50} / \theta_{\mathrm{E}}\right)$; this is seen for both dark matter and baryonic components in similar fashion.

In order to study the correlation between dark matter and baryonic slopes in the strong lensing region, Fig. [6]shows the projected baryonic slope $s_{\mathrm{b}}$ as a function of dark matter slope $s_{\mathrm{dm}}$ for the galaxy sample with $z_{\mathrm{d}}=0.2$ and $z_{\mathrm{s}}=1.5$. In fact a strong correlation between the two slopes is found for all of our samples with different $z_{\mathrm{d}}-z_{\mathrm{s}}$ combinations. This is a consequence of the strong interplay between dark matter and baryons in the central regions of galaxies.

The dependencies of the curvature parameters, as presented in Fig. 5] also exhibit similar distributions between dark matter and baryons. Once again, as the strong lensing region "moves out" (i.e., $\theta_{f 50} / \theta_{\mathrm{E}}$ decreasing), a slight increase (with concave curvature) followed by a decreasing trend (to becoming convex) develops from subgroup IV (green) to I (red), to II (blue) and to III (orange).

Figures 4 and 5 indicate the general trend of the (central and logarithmic) surface density profiles of both dark matter and baryonic distributions: with an increasing radius from the centre of a galaxy, a shallower and concave-upward profile in the inner region (probed by strong lensing of subgroup IV in green) is followed by a much steeper profile at intermediate radii (now probed by strong lensing of subgroup I in red and II in blue), and eventually is turning into a (slightly) shallower profile again with convex curvature at larger radii (now probed by strong lensing of subgroup III in green). Strong lensing therefore probes different regions of such matter density distributions. In a way, the distinctive profiles shown in Fig. 3 of galaxies in the four different subgroups are actually "snapshots" taken at different parts of the density distributions.

\subsection{Total density profiles}

We next present the results of the total (surface) density profiles. Fig. 7 shows the distributions of $s$ versus $\theta_{f 50} / \theta_{\mathrm{E}}$ (top left), $s$ versus $f_{\mathrm{dm}}\left(\theta_{\mathrm{E}}\right)$ (top right), $\xi$ versus $\theta_{f 50} / \theta_{\mathrm{E}}$ (bottom left) and $\xi$ versus $f_{\mathrm{dm}}\left(\theta_{\mathrm{E}}\right)$ (bottom right). Compared with Fig. 4]and [5 the distributions of the total density profile parameters have maintained the trends that are seen for both the dark matter and baryonic components.

Strong gravitational lensing probes different parts of the total density profile. This is the direct reason why we see a collection of various profile behaviours. Among the four subgroups, the most massive subgroup - the one with shallower and convex density profiles (III, in orange) - have the smallest $\theta_{f 50} / \theta_{\mathrm{E}}$. In particular their $\theta_{f 50} \lesssim 0.5 \theta_{\mathrm{E}}$ indicates that their dark matter-baryon interception radii are located within the inner boundaries of the strong lensing regions. As a result, the total density profile measured within the strong lensing regions is strongly dominated by dark matter, 


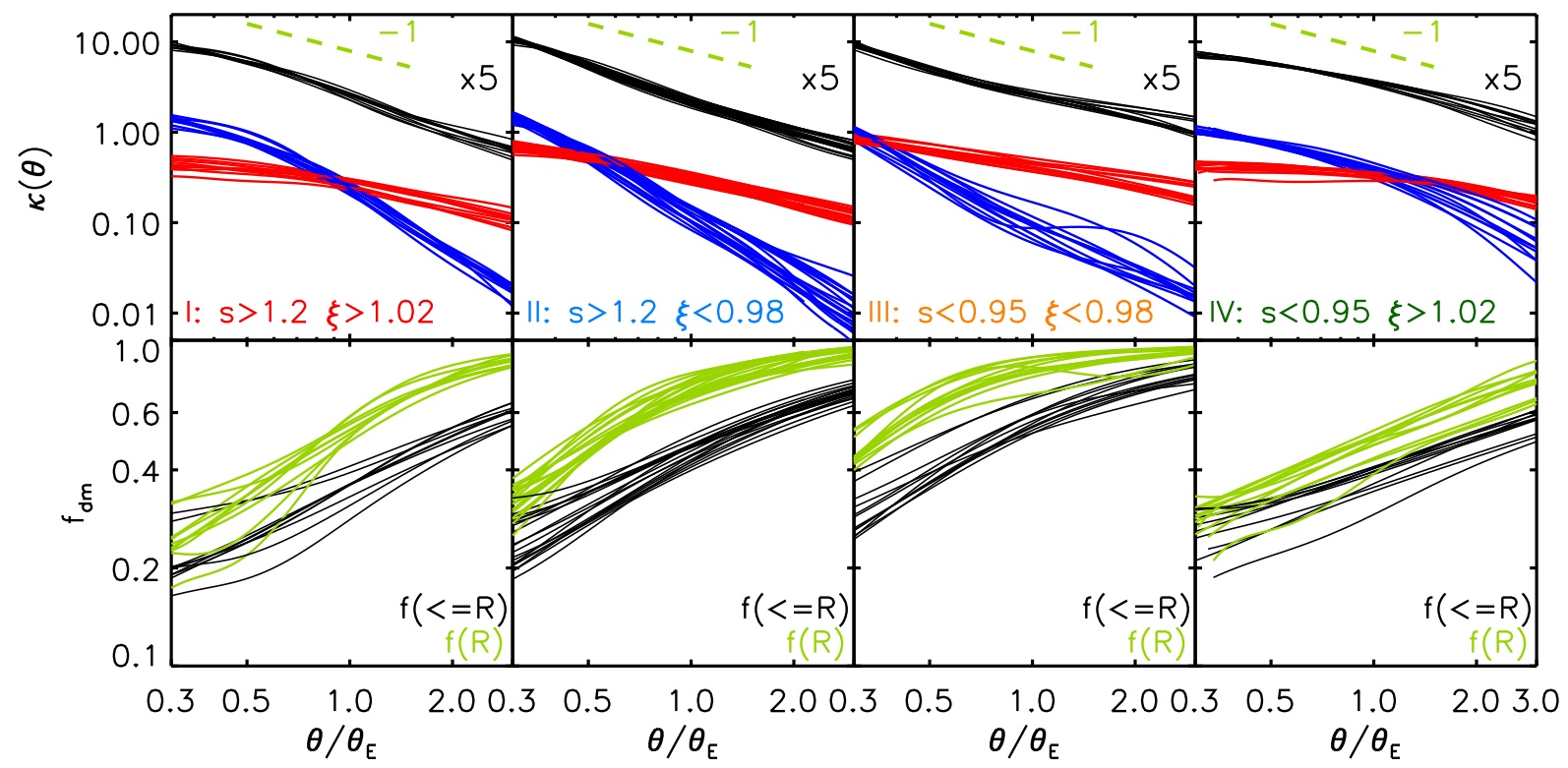

Figure 3. From each subgroup of the lens sample used in Fig. 1 ten typical surface density profiles and projected dark matter fraction distributions are shown. In the top panels the black curves show the total surface density distribution $\kappa(\theta)$, scaled up by a factor of 5 for clarity; the red and blue curves represent profiles of the projected dark matter $\kappa(\theta) f_{\mathrm{dm}}(\theta)$ and projected baryonic mass $\kappa(\theta)\left[1-f_{\mathrm{dm}}(\theta)\right]$, respectively; the dashed green line indicates the logarithmic slope of $s=1$ from $0.5 \theta_{\mathrm{E}}$ to $1.5 \theta_{\mathrm{E}}$. In the bottom panels, the distributions of the cumulative and local dark matter fraction $f_{\mathrm{dm}}(\leqslant \theta)$ and $f_{\mathrm{dm}}(\theta)$ are given in black and green, respectively.
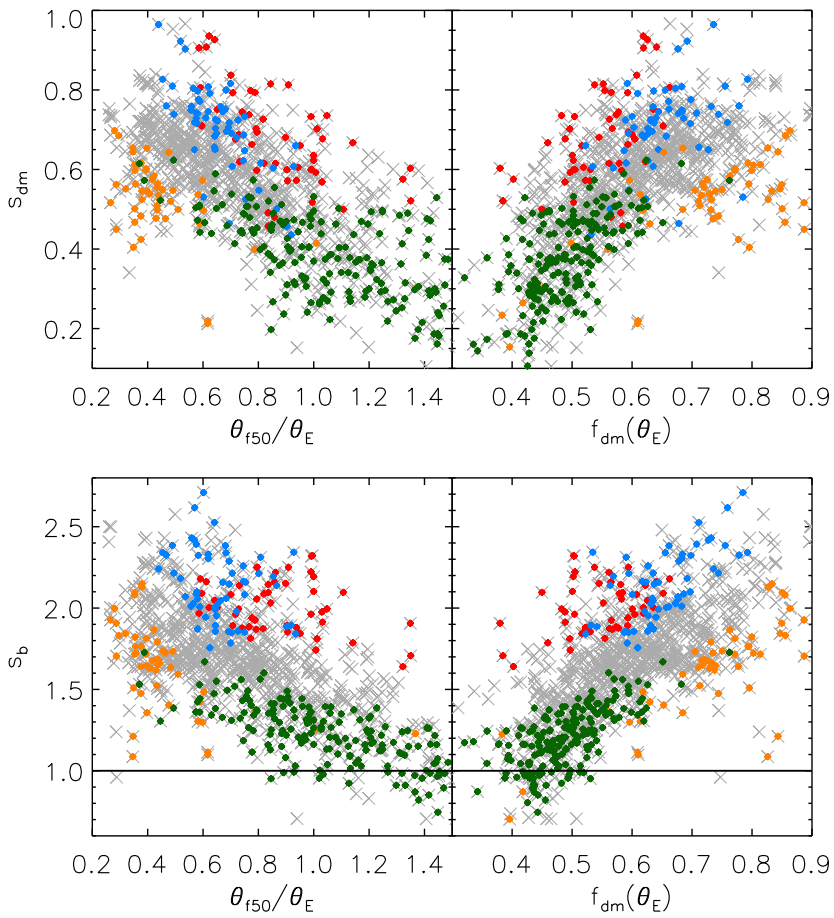

Figure 4. Top left: dark matter slope $s_{\mathrm{dm}}$ versus $\theta_{f 50} / \theta_{\mathrm{E}}$. Top right: $s_{\mathrm{dm}}$ versus $f_{\mathrm{dm}}\left(\theta_{\mathrm{E}}\right)$. Bottom left: baryonic slope $s_{\mathrm{b}}$ versus $\theta_{f 50} / \theta_{\mathrm{E}}$. Bottom right: $s_{\mathrm{b}}$ versus $f_{\mathrm{dm}}\left(\theta_{\mathrm{E}}\right)$. The galaxy sample as well as the colour coding is the same as in Fig. 1

whose distribution is considerably shallower than isothermal (see Fig. 4). In comparison, subgroup II galaxies (in blue) have $\theta_{f 50} \gtrsim 0.5 \theta_{\mathrm{E}}$, i.e., the dark matter-baryon interception
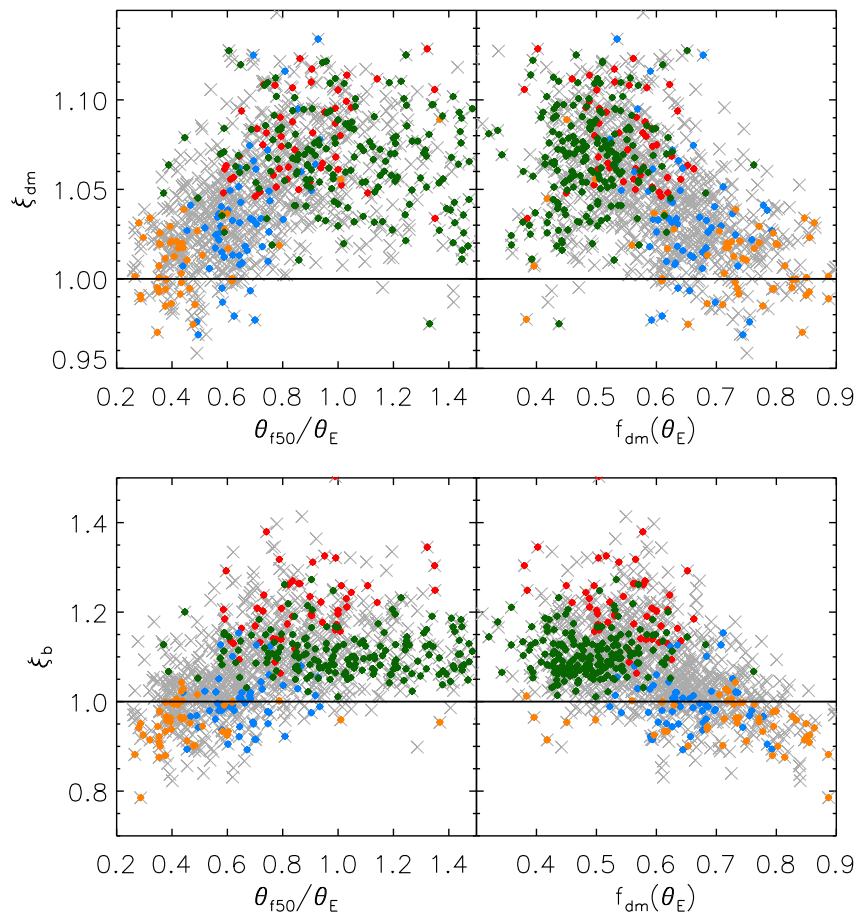

Figure 5. Top left: dark matter curvature $\xi_{\mathrm{dm}}$ versus $\theta_{f 50} / \theta_{\mathrm{E}}$. Top right: $\xi_{\mathrm{dm}}$ versus $f_{\mathrm{dm}}\left(\theta_{\mathrm{E}}\right)$. Bottom left: baryonic curvature $\xi_{\mathrm{b}}$ versus $\theta_{f 50} / \theta_{\mathrm{E}}$. Bottom right: $\xi_{\mathrm{b}}$ versus $f_{\mathrm{dm}}\left(\theta_{\mathrm{E}}\right)$. The galaxy sample as well as the colour coding is the same as in Fig. 1.

radii now exceed the inner radii of the strong lensing region, so that baryons can dominate the total density distribution at $\theta_{1}$ (see Fig. 3), resulting in the total slope $s$ 


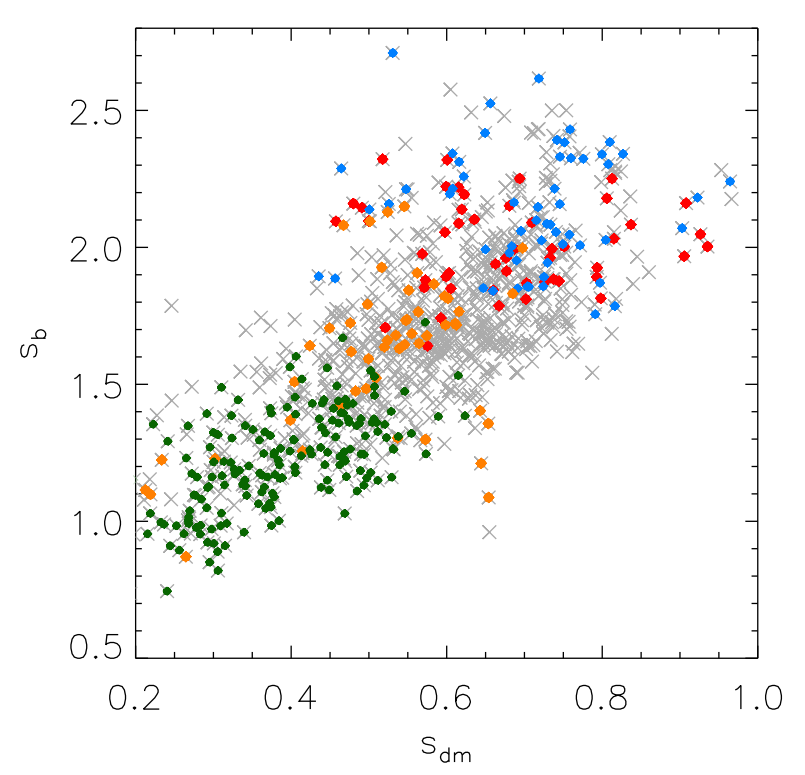

Figure 6. The projected baryonic slope $s_{\mathrm{b}}$ versus dark matter slope $s_{\mathrm{dm}}$, measured between $\theta_{1}=0.5 \theta_{\mathrm{E}}$ and $\theta_{2}=1.5 \theta_{\mathrm{E}}$. The galaxy sample and the colour coding are the same as in Fig. 1

steeper than isothermal. For both subgroups, a large fraction of the measured density distribution is dominated by dark matter; however, baryons contribute to a marked density upturn around the inner boundary of the strong lensing region, resulting in a convex curvature $(\xi<1)$ of the total profile.

In comparison, subgroup I (in red) and IV (in green) have even lower $f_{\mathrm{dm}}\left(\theta_{\mathrm{E}}\right)$ and larger $\theta_{f 50} / \theta_{\mathrm{E}}$, which means that baryons heavily dominate the strong lensing region in these cases. The behaviour of the total density profile, therefore, largely depends on how the baryonic matter is distributed. The strong lensing regions of subgroup IV galaxies are much closer to (the projected) galaxy centres than their subgroup I counterparts. This can be seen from larger $\theta_{f 50} / \theta_{\mathrm{E}}$ of the former than of the latter. As shown in Sect. 4.1, both dark and baryonic matter distributions are much shallower in the inner regions than at larger radii (see Fig. (4). As a result, the total density profiles of subgroup IV are shallower than of subgroup I galaxies; while both have concave curvatures in the strong lensing regions (i.e., $\xi_{\mathrm{dm}}>1$ and $\xi_{\mathrm{b}}>1$, see Fig. [5).

\section{THE DISTRIBUTIONS OF $\lambda$ AND THE CONSEQUENCE FOR $H_{0}$ MEASUREMENTS}

We have shown in Sect. 3 and 4 that galaxies can have a significant concavity/convexity compared to power law density profiles. Because of the existence of the MST, the lens modelling of these galaxies using a power-law density profile will yield a multiplicative bias $\lambda$ on $H_{0}$. We study hereafter the distributions of $\lambda$ resulting from the MST for the various samples of Illustris galaxies. Following our notation, the MST applied to the density profile $\kappa$ (via Eq. 1) such that the curvature parameter becomes unity, i.e., $\xi_{\lambda}=1$, yields
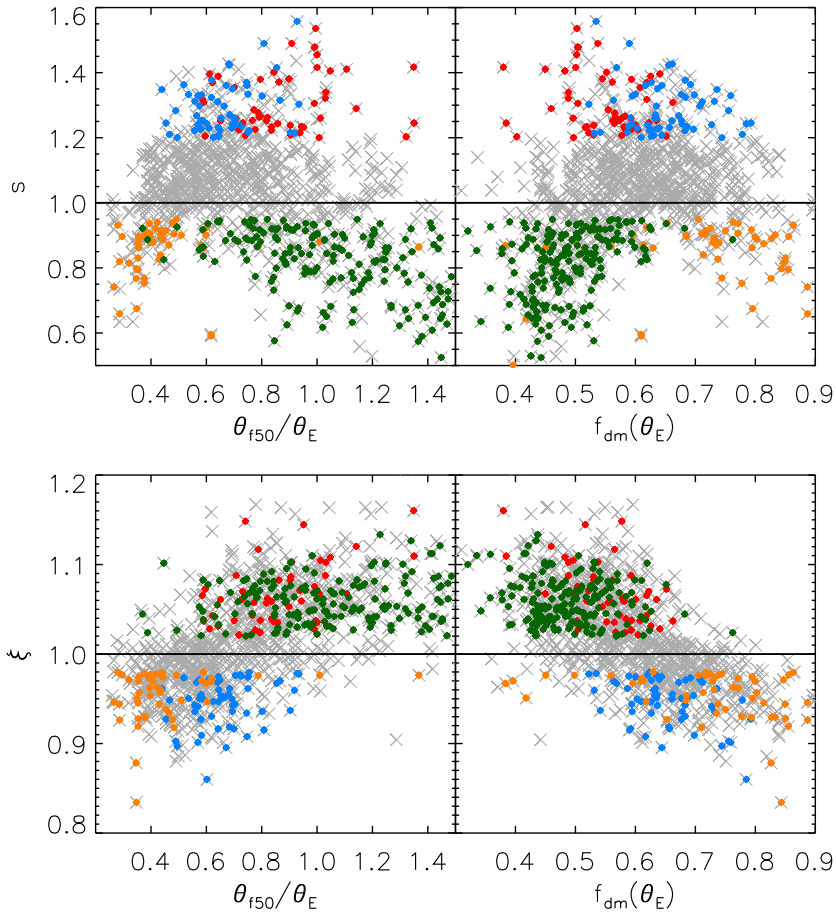

Figure 7. Top panels: $s$ versus $\theta_{f 50} / \theta_{\mathrm{E}}$ (left) and $s$ versus $f_{\mathrm{dm}}\left(\theta_{\mathrm{E}}\right)$ (right). Bottom panels: $\xi$ versus $\theta_{f 50} / \theta_{\mathrm{E}}$ (left) and $\xi$ versus $f_{\mathrm{dm}}\left(\theta_{\mathrm{E}}\right)$ (right). The galaxy sample and the colour coding are the same as in Fig. 1

a bias $\lambda$ and a transformed profile $\kappa_{\lambda}$ with a slope $s_{\lambda}$, while the MST on $\bar{\kappa}$ (via Eq. 11) is characterized by $\bar{\lambda}$ and $\bar{s}_{\lambda}$.

In Fig. 8 we present three sets of distributions, i.e., $\lambda$ and $\bar{\lambda}$ as a function of $\sigma_{\mathrm{SIE}}$ (left), $\theta_{\mathrm{E}} / \theta_{\text {eff }}$ (middle), and as a function of $f_{\mathrm{dm}}\left(\leqslant \theta_{\mathrm{E}}\right)$ (right), for the same galaxy sample as used before (where $z_{\mathrm{d}}=0.2$ and $z_{\mathrm{s}}=1.5$ ). We see that the distributions of $\lambda$ and $\bar{\lambda}$ span a wide range of values from 0 to 1.5 and exhibit a large scatter without a strong dependence on any of the observables above. Figures displayed in Appendix $\mathrm{C}$ show that similar distributions are also present for all other studied $z_{\mathrm{d}}-z_{\mathrm{s}}$ combinations. It is however noticeable that for galaxies with $200 \mathrm{~km} \mathrm{~s}^{-1}<\sigma_{\mathrm{SIE}}<300 \mathrm{~km} \mathrm{~s}^{-1}$, the mean multiplicative bias $\langle\lambda\rangle$ is generally close to unity with deviations not larger than $\sim 20 \%$ at lower $\sigma_{\text {SIE }}$, while for $\bar{\lambda}$ the mean deviation can be as large as $\sim 50 \%$. In all cases, an rms of $10 \%-30 \%$ is present. This emphasizes that the power-law assumption in lens modelling is highly questionable, as it will create non-negligible biases in the derived values of $H_{0}$. However, we note that the observed "time delay lenses" have generally large velocity dispersion $\left(\sigma_{\mathrm{SIE}} \gtrsim 250 \mathrm{~km} \mathrm{~s}^{-1}\right)$, for which $\lambda$ is close to unity on average, though with a $10 \%-20 \%$ scatter.

Interestingly, when correlating $\lambda$ with $s_{\lambda}$ (and $\bar{\lambda}$ with $\bar{s}_{\lambda}$ ), which is the "measured" mean slope using power-law lens models, we see a much clearer systematic trend with smaller scatters. This is shown in Fig. 9] where the distributions of $\lambda$ versus $s_{\lambda}$ (left) and $\bar{\lambda}$ versus $\bar{s}_{\lambda}$ (right) are presented. Such a tight correlation is also seen for all other samples with different $z_{\mathrm{d}}-z_{\mathrm{s}}$ combinations (see Fig. C2).

A striking result from Fig. 9 is that the $s_{\lambda}$ (and $\left.\bar{s}_{\lambda}\right)$ distribution of galaxies with $\lambda \approx 1$ (and $\bar{\lambda} \approx 1$ ) peaks at 


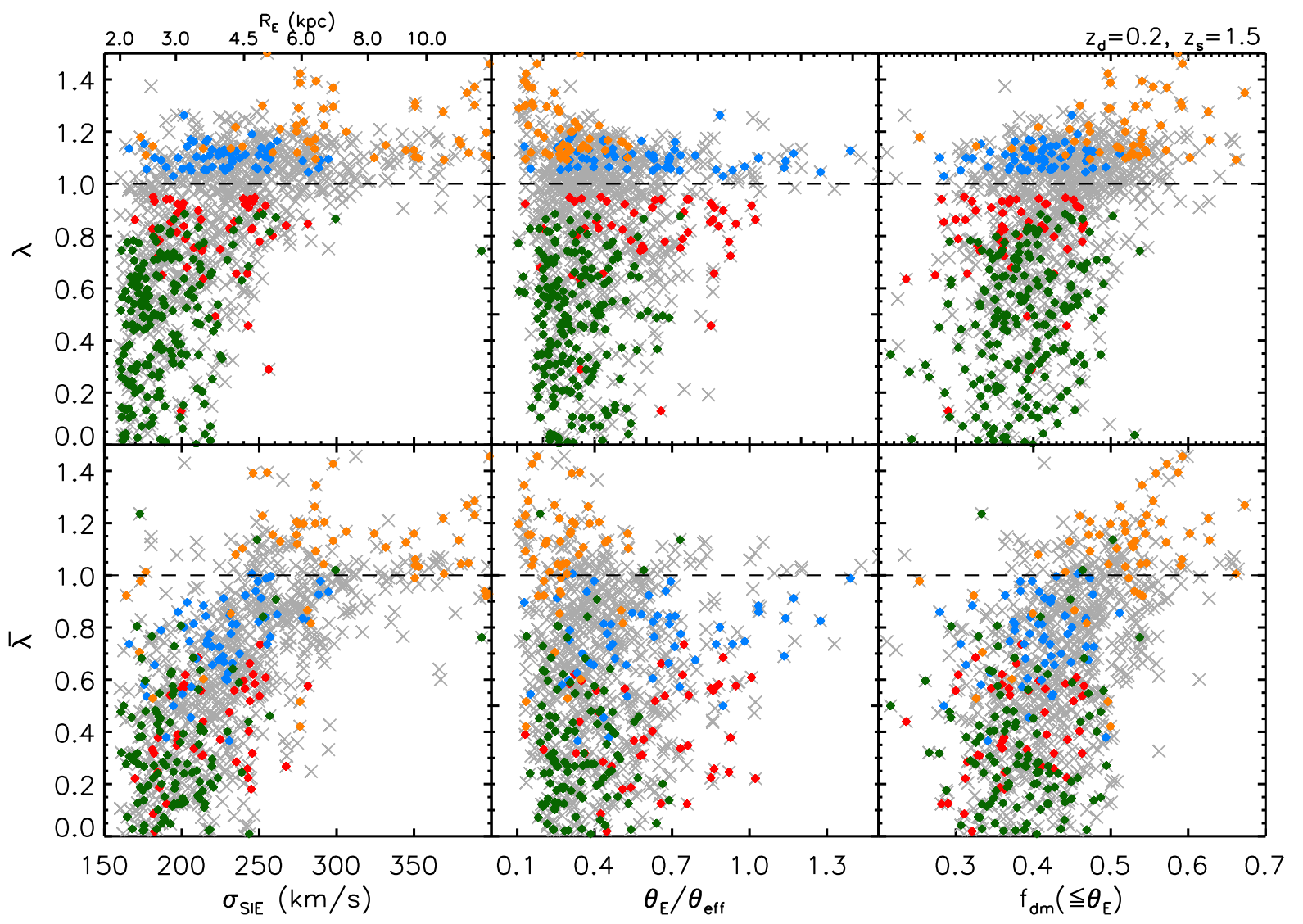

Figure 8. The distributions of $\lambda$ (top) and $\bar{\lambda}$ (bottom) versus $\sigma_{\mathrm{SIE}}$ (left), versus $\theta_{\mathrm{E}} / \theta_{\mathrm{eff}}$ (middle) and versus $f_{\mathrm{dm}}\left(\leqslant \theta_{\mathrm{E}}\right)$ (right). The sample as well as the colour coding is the same as in Fig. 1 Similar distributions are also seen for all other studied $z_{\mathrm{d}}-z_{\mathrm{s}}$ combinations. The upper axis of the top-left panel indicates the physical Einstein radius.

$s_{\lambda} \approx 1\left(\right.$ and $\left.\bar{s}_{\lambda} \approx 1\right)$; and the $\lambda($ and $\bar{\lambda})$ distribution of galaxies with $s_{\lambda} \approx 1$ (and $\bar{s}_{\lambda} \approx 1$ ) peaks at $\lambda \approx 1$ (and $\bar{\lambda} \approx 1)$. This could be potentially used to select time-delay samples which minimize the impact of the MST on $H_{0}$ under the power-law assumption.

We have further investigated such a possibility. For each of our lens samples, we have defined three subsamples that satisfy $(1) s_{\lambda} \in\left[1-\Delta_{s}, 1+\Delta_{s}\right] ;(2) \bar{s}_{\lambda} \in\left[1-\Delta_{s}, 1+\Delta_{s}\right]$ and (3) both $s_{\lambda} \in\left[1-\Delta_{s}, 1+\Delta_{s}\right]$ and $\bar{s}_{\lambda} \in\left[1-\Delta_{s}, 1+\Delta_{s}\right]$, respectively. The slope $\operatorname{span} \Delta_{s}$ is set to be 0.1 , i.e., the "measured" mean slopes (under the power-law assumption) are very close to isothermal. Table 2 summarizes the statistical properties of $\lambda$ and $\bar{\lambda}$ distributions for the subsamples. In all the cases using these subsamples, both the systematic deviation from unity $(\lesssim 5 \%)$ and the scatter $(\lesssim 10 \%)$ are much smaller than those for the general samples.

The statistical results presented above indicate that the power-law assumption will introduce non-negligible biases in $H_{0}$ measurements, even though adopting power-law lens models is a common practice. However, through a careful sample selection, e.g., selecting galaxies that have larger velocity dispersions (i.e., large angular separations of the multiple images) or those which have the "measured" profiles close to isothermal under the power-law assumption, the use of simple power-law models may yield estimates of $H_{0}$ which are biased only at the few percent level - compared to a much larger bias from the total sample studied here. This work also shed light on such a possibility in this direction.

\section{DISCUSSION AND CONCLUSIONS}

The central regions of massive galaxies (i.e. typically the inner $5-10 \mathrm{kpc}$ ) contain dark and baryonic matter in roughly similar amounts. Although both components follow different density distributions, it has been found that the total density profile in that region is well approximated by a single power law (e.g., Koopmans et al. 2006, Gavazzi et al, 2007). This has motivated the use of the power-law model $\rho \propto r^{-\gamma^{\prime}}$ as a generic model for numerous galaxy-scale strong lensing studies. However, as emphasized by Schneider \& Sluse (2013), a mass distribution $\kappa(\theta)$ can be transformed into an approximate power law by means of an MST, i.e. $\kappa_{\lambda}(\theta)=\lambda \kappa(\theta)+(1-\lambda)$, even if the true mass profile shows considerable curvature. Under a MST, none of the lensing observables are modified except for the product of the Hubble constant and the time delay $H_{0} \Delta t$, which is scaled by the same factor $\lambda$ as the one characterizing the MST. By assuming a power-law profile, one "artificially" breaks the MSD and derives a biased estimate of $H_{0}$. In addition, the use of a single quantity, i.e., the logarithmic slope $\gamma^{\prime}$, to characterize the density profile of galaxies may be misleading, since the intrinsic density does not follow an exact power 

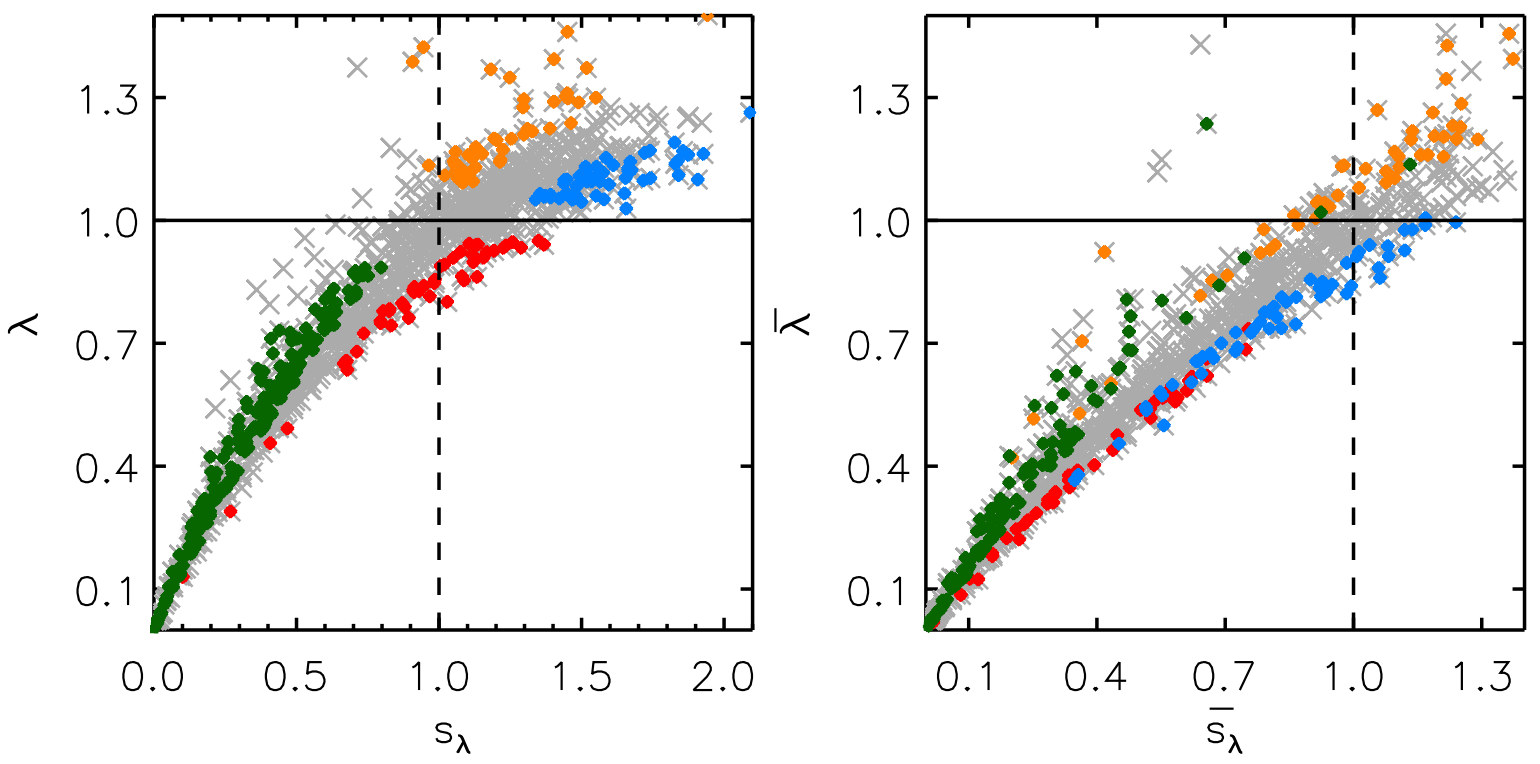

Figure 9. The distributions of $\lambda$ versus $s_{\lambda}$ (left) and $\bar{\lambda}$ versus $\bar{s}_{\lambda}$ (right). The sample as well as the colour coding is the same as in Fig. 1 Similar distributions are also seen for all other studied $z_{\mathrm{d}}-z_{\mathrm{s}}$ combinations (given in the Appendix C). The solid lines indicate where $\lambda=1$ and where $\bar{\lambda}=1$; while the dashed lines indicate where $s_{\lambda}=1$ and where $\bar{s}_{\lambda}=1$.

Table 2. A summary of the statistical properties of $\lambda$ and $\bar{\lambda}$ for galaxy subsamples that satisfy $s_{\lambda} \in[0.9,1.1]$ and/or $\bar{s}_{\lambda} \in[0.9,1.1]$ :

\begin{tabular}{|c|c|c|c|c|c|c|}
\hline Sample sets & & $z_{\mathrm{s}}=1.5$ & & & $z_{\mathrm{d}}=0.6$ & \\
\hline Redshifts & $z_{\mathrm{d}}=0.2$ & $z_{\mathrm{d}}=0.4$ & $z_{\mathrm{d}}=0.8$ & $z_{\mathrm{s}}=1.0$ & $z_{\mathrm{s}}=1.5$ & $z_{\mathrm{s}}=3.0$ \\
\hline \multicolumn{7}{|c|}{ Subsample I: $s_{\lambda} \in[0.9,1.1]$} \\
\hline Number of galaxy projections & 142 & 210 & 174 & 146 & 223 & 296 \\
\hline Mean $\lambda$ & 1.00 & 0.98 & 0.97 & 0.97 & 0.97 & 0.97 \\
\hline Median $\lambda$ & 0.99 & 0.97 & 0.96 & 0.95 & 0.96 & 0.97 \\
\hline Standard deviation $\sigma_{\lambda}$ & 0.11 & 0.09 & 0.10 & 0.12 & 0.10 & 0.08 \\
\hline \multicolumn{7}{|c|}{ Subsample II: $\bar{s}_{\lambda} \in[0.9,1.1]$} \\
\hline Number of galaxy projections & 110 & 167 & 148 & 102 & 184 & 278 \\
\hline Mean $\bar{\lambda}$ & 0.98 & 0.98 & 0.97 & 0.96 & 0.97 & 0.98 \\
\hline Median $\bar{\lambda}$ & 0.98 & 0.98 & 0.95 & 0.96 & 0.97 & 0.97 \\
\hline Standard deviation $\sigma_{\bar{\lambda}}$ & 0.08 & 0.09 & 0.11 & 0.09 & 0.10 & 0.14 \\
\hline \multicolumn{7}{|c|}{ Subsample III: $s_{\lambda} \in[0.9,1.1]$ and $\bar{s}_{\lambda} \in[0.9,1.1]$} \\
\hline Number of galaxy projections & 19 & 36 & 21 & 19 & 37 & 63 \\
\hline Mean $\lambda$ & 1.04 & 1.02 & 1.04 & 1.06 & 1.01 & 1.01 \\
\hline Median $\lambda$ & 1.03 & 1.02 & 1.01 & 1.04 & 1.02 & 1.01 \\
\hline Standard deviation $\sigma_{\lambda}$ & 0.06 & 0.06 & 0.10 & 0.08 & 0.05 & 0.07 \\
\hline Mean $\bar{\lambda}$ & 1.02 & 1.02 & 1.04 & 1.06 & 1.01 & 1.02 \\
\hline Median $\bar{\lambda}$ & 1.01 & 1.01 & 1.02 & 1.04 & 1.01 & 1.01 \\
\hline Standard deviation $\sigma_{\bar{\lambda}}$ & 0.05 & 0.06 & 0.12 & 0.08 & 0.07 & 0.08 \\
\hline
\end{tabular}

law. Therefore, such a slope depends on the radius, and its comparison among galaxies depends on the range of galactocentric radii over which it is measured [note that Dutton \& Treu 2014 suggests the use of a mass-weighted slope to alleviate the problem].

In this paper, we have used a statistical sample of mock lensing galaxies from the first high-resolution cosmologicalscale hydrodynamic simulation - the Illustris Project (Vogelsberger et al. 2014, see also Vogelsberger et al. 2014; Genel et al. 2014; Nelson et al. 2015) - to test the validity of the power-law assumption for the (central) surface density distribution of strong gravitational lenses. The simulation reproduces extremely well numerous observational properties of real galaxies. In particular, we find that the cumulative dark matter fraction $f_{\mathrm{dm}}\left(\leqslant \theta_{\mathrm{E}}\right)$ of the simulated lensing galaxies ranges from $30 \%$ to $70 \%$, in good agreement with observations (Treu \& Koopmans 2004; Koopmans et al. 2006). The average isothermality of the profiles derived for the simulated galaxies is also in agreement with various observational studies (e.g., Rusin et al. 2003; Auger et al. 2010; Sonnenfeld et al. 2013). A detailed analysis of the 3d density profile in the inner regions of Illustris lenses will be presented in a forthcoming paper (Xu et al., in preparation).

From the mock Illustris lensing galaxies at each of the studied lens redshifts below $z=1$, we have taken a sample of about 400 plausible lens galaxies, which have central velocity dispersion $\sigma \geqslant 160 \mathrm{~km} \mathrm{~s}^{-1}$ and are "observed" through their three independent projections, yielding a sample of more 
than 1000 projected density profiles. The slopes and curvatures of the projected density profiles in regions probed by strong lensing (typically between 0.5 and 1.5 angular Einstein radii $\theta_{E}$ ) have been measured. We have transformed these profiles into (local) power laws via an MST and derived the distribution of the multiplicative bias $\lambda$ on $H_{0}$ implied by a power-law assumption of the density profile.

Our main findings are as follows:

- The total surface density profile in the projected central regions of galaxies depends on radius, showing deviations from a power law following some generic trends. At smaller radii the profiles are shallow and concave upward. With increasing radius, the profiles gradually steepen, and eventually at larger radii they become marginally shallower and attain convex curvatures. The projected density distributions of both dark and baryonic matter follow such a general trend, while the latter is more concentrated (and steeper) than the former. Good correlations exist between the dark matter and baryonic density slopes throughout the projected radii and redshifts studied in this work, indicating the strong interplay between dark matter and baryons in central regions of galaxies.

- In the region probed by strong lensing, the (projected) total density profile deviates significantly (by up to 15\%) from an exact power law, with a variety of slopes and curvatures. The main reason for the diverse measurements of the profile parameters is that strong lensing probes different parts of this density distribution; the measured slopes and curvatures depend on the relative contribution of dark and baryonic matter in regions where the measurements are made. In general dark matter dominates the strong lensing regions of massive galaxies, which therefore tend to have mean profiles (in the strong lensing region) shallower than isothermal. In contrast, lower-mass galaxies have nonnegligible baryonic components within their strong lensing regions; the shapes of the mean total density profiles are thus largely determined by the baryonic distributions.

- The curvature of the (logarithmic) density profile is considerable and produces a non-negligible bias in the measured $H_{0}$, if only lensing information is used. The statistical distribution of $\lambda$ (and $\bar{\lambda}$ ) derived from the Illustris strong lens sample spans from 0 to 1.5 and exhibits large scatter without a strong dependence on observationally-constrained galaxy properties, such as $\sigma_{\mathrm{SIE}}, \theta_{\mathrm{E}} / \theta_{\text {eff }}$ and $f_{\mathrm{dm}}\left(\leqslant \theta_{\mathrm{E}}\right)$. In particular, the mean deviation of $\lambda$ (and $\bar{\lambda}$ ) from unity can be as large as $20 \%-50 \%$ with a scatter of $10 \%-30 \%(\mathrm{rms})$ for galaxies with $\sigma_{\mathrm{SIE}}=200 \mathrm{~km} \mathrm{~s}^{-1}-300 \mathrm{~km} \mathrm{~s}^{-1}$. We note, however, that galaxies with derived slopes (under the powerlaw assumption) close to isothermal exhibit much smaller systematic deviation of $\lesssim 5 \%$ from unity with a scatter of $\lesssim 10 \%$ (rms). This could potentially be used to form a reliable sample of lensing galaxies for $H_{0}$ measurements in the upcoming big data era.

We would like to stress that by finding an MST which approximates $\kappa_{\lambda}$ as closely as possible by a power law, we in essence approximate $\kappa$ locally by a power law plus a uniform mass sheet. However, this is purely a mathematical description; this uniform mass sheet is not assigned any physical meaning. In particular, this mass sheet cannot (or only partly) be interpreted as a convergence due to foreground and background material along the line of sight. The latter contribution to the convergence can be estimated/constrained using cosmological simulations and/or through detailed studies of the lens environment or weak lensing (e.g., Suvu et al. 2010, 2013). Furthermore, even if a lens is perfectly fitted with $\kappa$ being a power law plus uniform mass sheet, then the same is true for all $\kappa_{\lambda}$ through MST. Hence, $\lambda$ remains undetermined.

It is also worth noting that one may try out different "realistic" model profiles for the mass distribution of lenses, such as the composite ones used in Schneider \& Sluse (2013) or in Suyu et al. (2014), in order to assess the systematics due to the MST. However the principal problem remains: what is the possible range of "realistic" mass models? In other words, if a (possible composite) model $\kappa$ fits the data, how can one rule out that the corresponding model $\kappa_{\lambda}$ (with, say, $0.85 \leqslant \lambda \leqslant 1.15$ ) is not "realistic"? The various density profiles shown in Fig. 3 indicate that real galaxies most likely exhibit a fairly large range of density profiles, which makes the classification of models profiles into "realistic" and less realistic ones fairly difficult, if not all impossible.

Thereby biased estimates of $H_{0}$ are not fundamentally due to the power-law assumption of the lens model but are caused by the MSD itself, whose impact cannot be eliminated simply by studying the properties of material in the surrounding light cone, or through the adoption of more sophisticated lens models even when extra constraints are available from extended image configurations. In order to obtain a reliable estimate of $H_{0}$ using gravitational lensing, one must first break the MSD.

One possibility of doing so is to use the fact that MST also modifies image magnification $\mu \rightarrow \mu / \lambda^{2}$. If the source luminosity, and thus the absolute magnification, is known $\lambda$ can be fixed. For AGN as sources, as shown by Bauer et al. (2012), the variability-luminosity relation can be used to estimate source luminosities but only in a statistical way with large scatter. Alternatively, if Type Ia supernovae are gravitationally lensed (Chornock et al. 2013; Quimby et al. 2013, 2014), direct measurements of $\mu$ would be possible due to their nature as approximate standard candles. In such cases, the MSD can also be broken and the multiply-lensed Type Ia supernovae become ideal laboratories to study the true lens mass distribution as well as cosmological parameters (Kolatt \& Bartelmann 1998; Oguri \& Kawano 2003; Bolton \& Burles 2003).

A second way to break the MSD is to include independent information about the lens mass distribution, e.g., from stellar dynamics (e.g., Barnabè \& Koopmans 2007; Barnabè et al. 2009, 2011; Treu \& Koopmans 2002; Suvu et al. 2010, 2013, 2014). The problem is that the current accuracy (typically 10\%) in the velocity dispersion measurements may not yet be sufficient to break the MSD, not to mention the uncertainty due to the anisotropy of stellar orbits, which can lead to a systematic error on the slope at a level of $\sim 5 \%$ (Agnello et al. 2013). Further investigations in this regard were not included in this analysis but are greatly encouraged.

\section{ACKNOWLEDGEMENTS}

The authors would like to thank Stefan Hilbert, Sherry Suyu, Malte Tewes, James Nightingale and an anonymous 
referee for very useful comments. DDX thanks the HITS fellowship. DS acknowledges support from a Back to Belgium grant from the Belgian Federal Science Policy (BELSPO), and partial funding from the Deutsche Forschungsgemeinschaft, reference SL172/1-1. PS and VS acknowledge support by the DFG through Transregio 33, "The Dark Universe". VS also acknowledges support by the European Research Council under ERC-StG EXAGAL-308037. LH acknowledges support from NASA grant NNX12AC67G and NSF grant AST-1312095. 

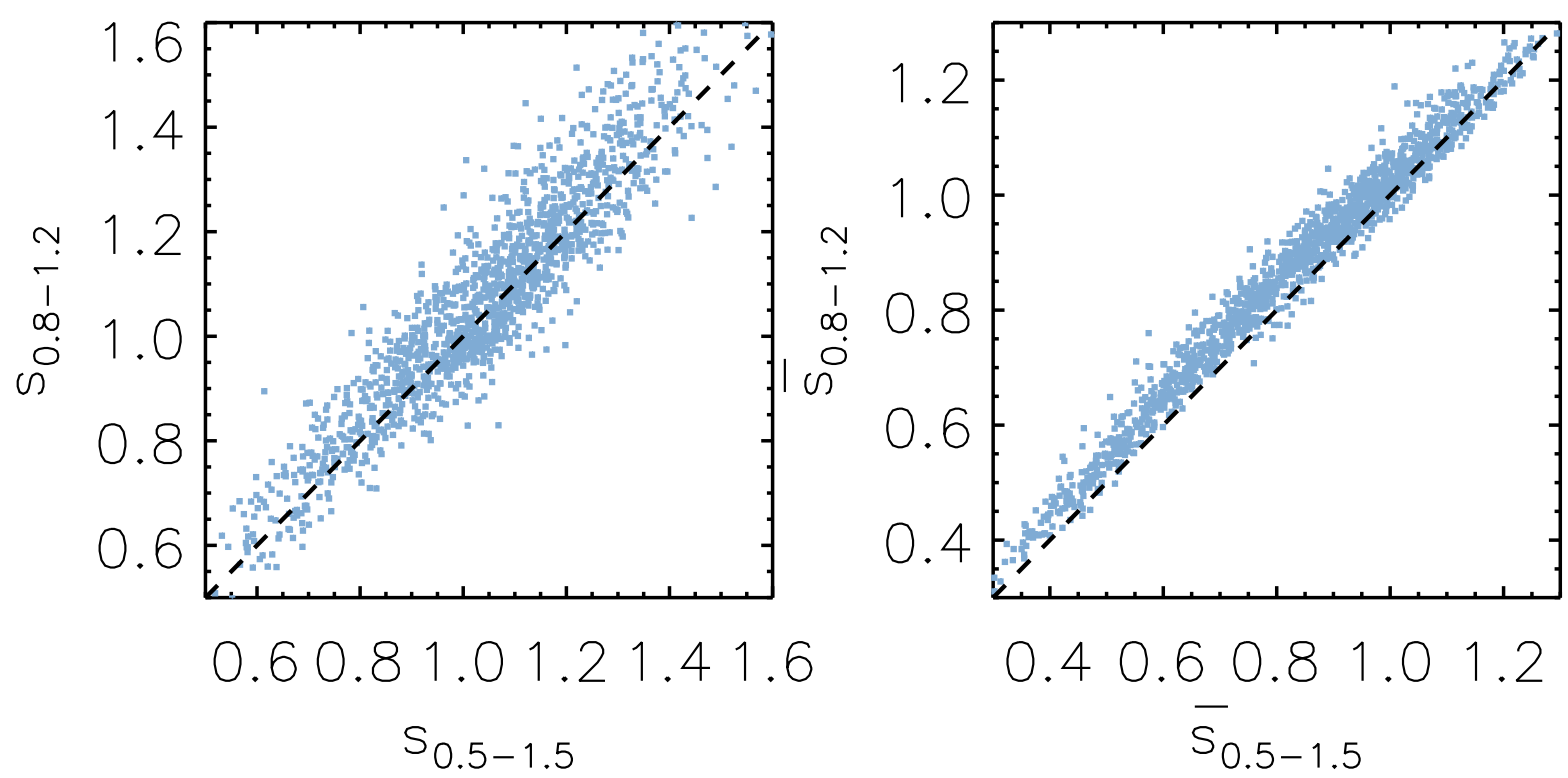

Figure A1. The slopes defined within different radial ranges are compared for the lens sample with $z_{\mathrm{d}}=0.6$ and $z_{\mathrm{s}}=1.5$. On the left, the slopes of the local convergence $\kappa(\theta)$ measured between $\theta_{1}=0.8 \theta_{\mathrm{E}}$ and $\theta_{2}=1.2 \theta_{\mathrm{E}}$ are plotted versus those measured between $\theta_{1}=0.5 \theta_{\mathrm{E}}$ and $\theta_{2}=1.5 \theta_{\mathrm{E}}$; on the right the relation of the cumulative distribution $\bar{\kappa}(\leqslant \theta)$ is given.

\section{APPENDIX A: VARYING THE RANGE OF THE STRONG LENSING REGION}

Different angular ranges $\left[\theta_{1}, \theta_{2}\right]$ will not result in exactly the same transformed power-law profiles and the associated $\lambda \mathrm{s}$. It is therefore interesting to consider if our final statistical results strongly depend on the choice of the image range. In Fig. A1, we present the slopes measured between $\theta_{1}=0.5 \theta_{\mathrm{E}}$ and $\theta_{2}=1.5 \theta_{\mathrm{E}}$ and between $\theta_{1}=0.8 \theta_{\mathrm{E}}$ and $\theta_{2}=1.2 \theta_{\mathrm{E}}$. The corresponding $\lambda$ distributions are shown in Fig. A2 As can be seen, although measurements in these two cases are not exactly the same on a one-to-one basis, the statistical distributions of $\lambda$ are independent of the image range sets. We have, therefore, set $\theta_{1}=0.5 \theta_{\mathrm{E}}$ and $\theta_{2}=1.5 \theta_{\mathrm{E}}$ to calculate the relevant quantities throughout the paper.

\section{APPENDIX B: DENSITY PROFILES}

In this appendix, we show the density profiles of our galaxy samples. Fig. B1 presents the shape parameter distribution in the $\bar{s}-\bar{\xi}$ plane (see Sect. 2 for definition). Fig. B2 shows the surface density profiles of galaxies from each subgroup of the lens sample presented in Fig. 3, which are now normalized by galaxy effective radii $\theta_{\text {eff }}$.

\section{APPENDIX C: THE $\lambda$ DISTRIBUTIONS OF ALL LENS SAMPLES}

In this appendix, we present the distributions of $\lambda$ (and $\bar{\lambda}$ ) as a function of $\sigma_{\mathrm{SIE}}$ and of the "measured slope" $s_{\lambda}$ (and $\bar{s}_{\lambda}$ ) for our lens samples with different sets of $z_{\mathrm{d}}$ and $z_{\mathrm{s}}$ combinations. 

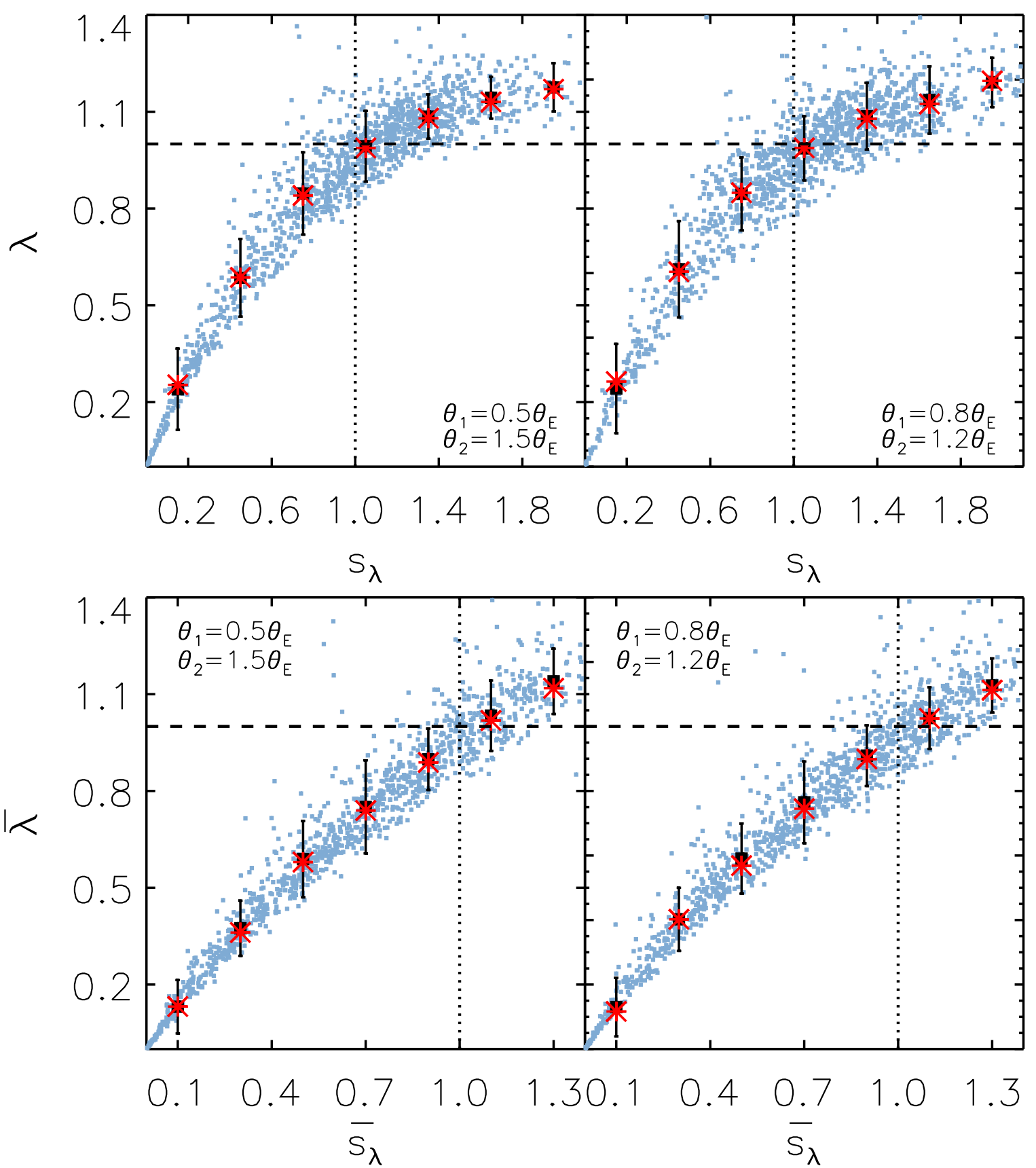

Figure A2. The distributions of $\lambda$ versus $s_{\lambda}$ (top panels) and $\bar{\lambda}$ versus $\bar{s}_{\lambda}$ (bottom panels) for the lens sample with $z_{\mathrm{d}}=0.6$ and $z_{\mathrm{s}}=1.5$ : the left-hand side panel shows the distributions calculated assuming $\theta_{1}=0.5 \theta_{\mathrm{E}}$ and $\theta_{2}=1.5 \theta_{\mathrm{E}}$; for comparison, the results for which $\theta_{1}=0.8 \theta_{\mathrm{E}}$ and $\theta_{2}=1.2 \theta_{\mathrm{E}}$ are adopted are given on the right-hand side. On top of the scattered data (blue dots), the black squares (and red stars) with the error bars indicate the mean (and median) and its standard deviation within each bin. The dashed lines indicate where $\lambda=1$ and where $\bar{\lambda}=1$; while the dotted lines indicate where $s_{\lambda}=1$ and where $\bar{s}_{\lambda}=1$. 


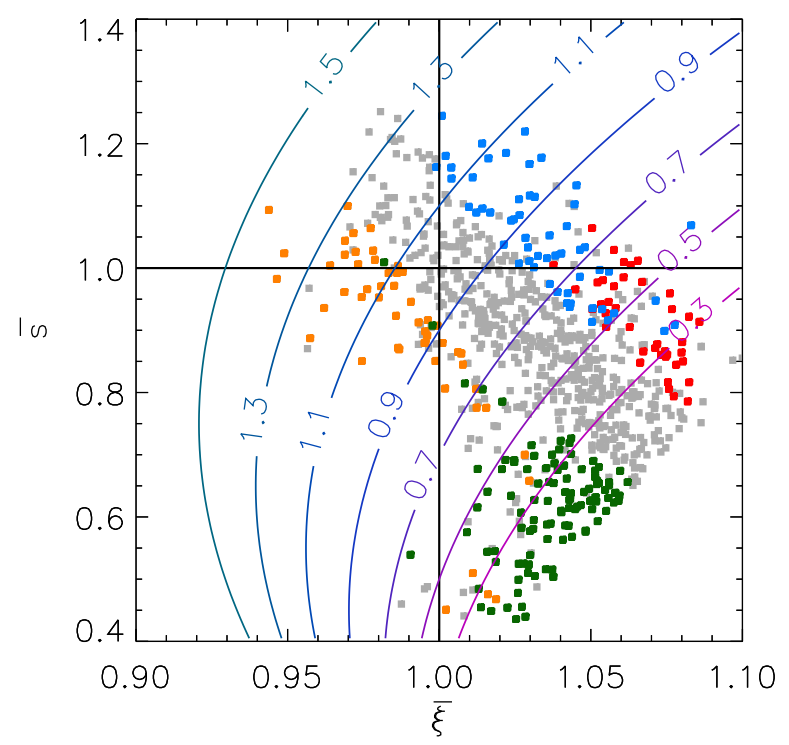

Figure B1. The $\bar{s}-\bar{\xi}$ distribution of the selected lensing galaxies at $z_{\mathrm{d}}=0.2$, assuming $z_{\mathrm{s}}=1.5$. Four colours represent four subgroups divided according to $s$ and $\xi$ of the local convergence distribution $\kappa(\theta)$ : subgroup I (red) are defined as galaxies with $s>1.2, \xi>1.02$; subgroup II (blue) are those with $s>1.2, \xi<0.98$; subgroup III (orange) represents galaxies with $s<0.95, \xi<0.98$; and subgroup IV (green) are those with $s<0.95, \xi>1.02$. Contours indicate where the transformed slopes $\bar{s}_{\lambda}=[0.3,0.5,0.7,0.9,1.1,1.3,1.5,1.7]$.

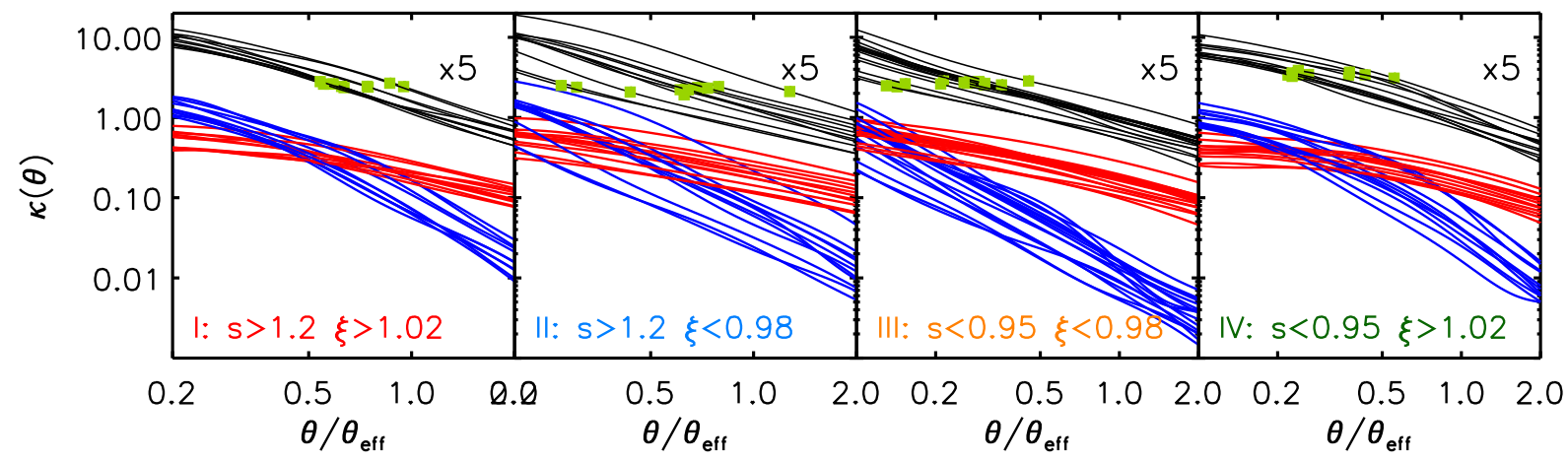

Figure B2. The surface density profiles of galaxies from each subgroup of the lens sample presented in Fig. 3 are shown as normalized by galaxy effective radii $\theta_{\text {eff }}$. The black curves show the total surface density distribution $\kappa(\theta)$, scaled up by a factor of 5 for clarity; the red and blue curves represent profiles of the projected dark matter $\kappa(\theta) f_{\mathrm{dm}}(\theta)$ and projected baryonic mass $\kappa(\theta)\left(1-f_{\mathrm{dm}}(\theta)\right)$, respectively; green dots indicate where the Einstein radii are with respect to the effective radii. 

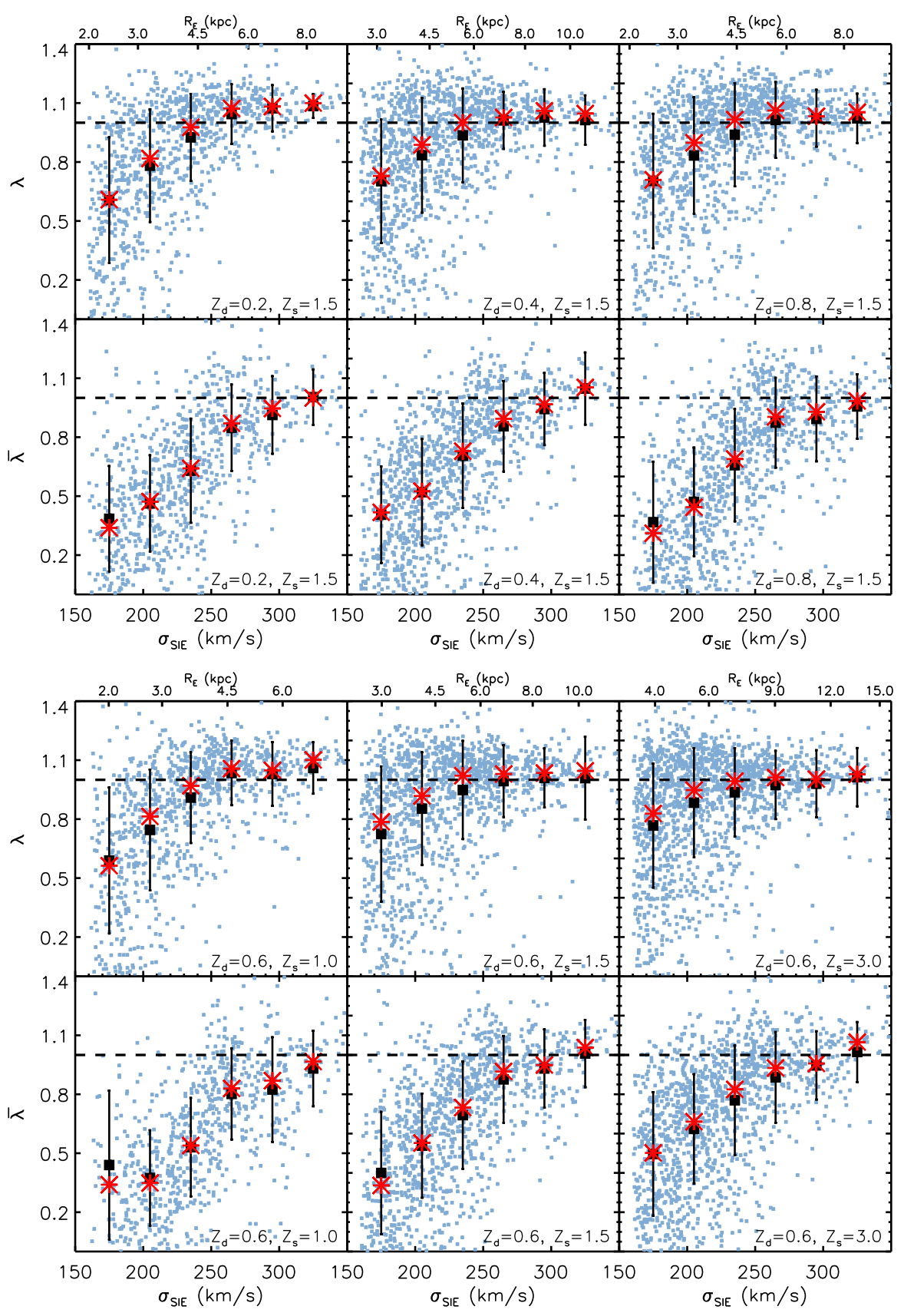

Figure C1. Distributions of $\lambda$ (and $\bar{\lambda}$ ) versus $\sigma_{\mathrm{SIE}}$ for all six samples with $z_{\mathrm{d}}$ and $z_{\mathrm{s}}$ indicated in the panels. On top of the scattered data (blue dots), the black squares (and red stars) with the error bars indicate the mean (and median) and its standard deviation within each bin. The dashed lines indicate where $\lambda=1$ and where $\bar{\lambda}=1$; while the dotted lines indicate where $s_{\lambda}=1$ and where $\bar{s}_{\lambda}=1$. 

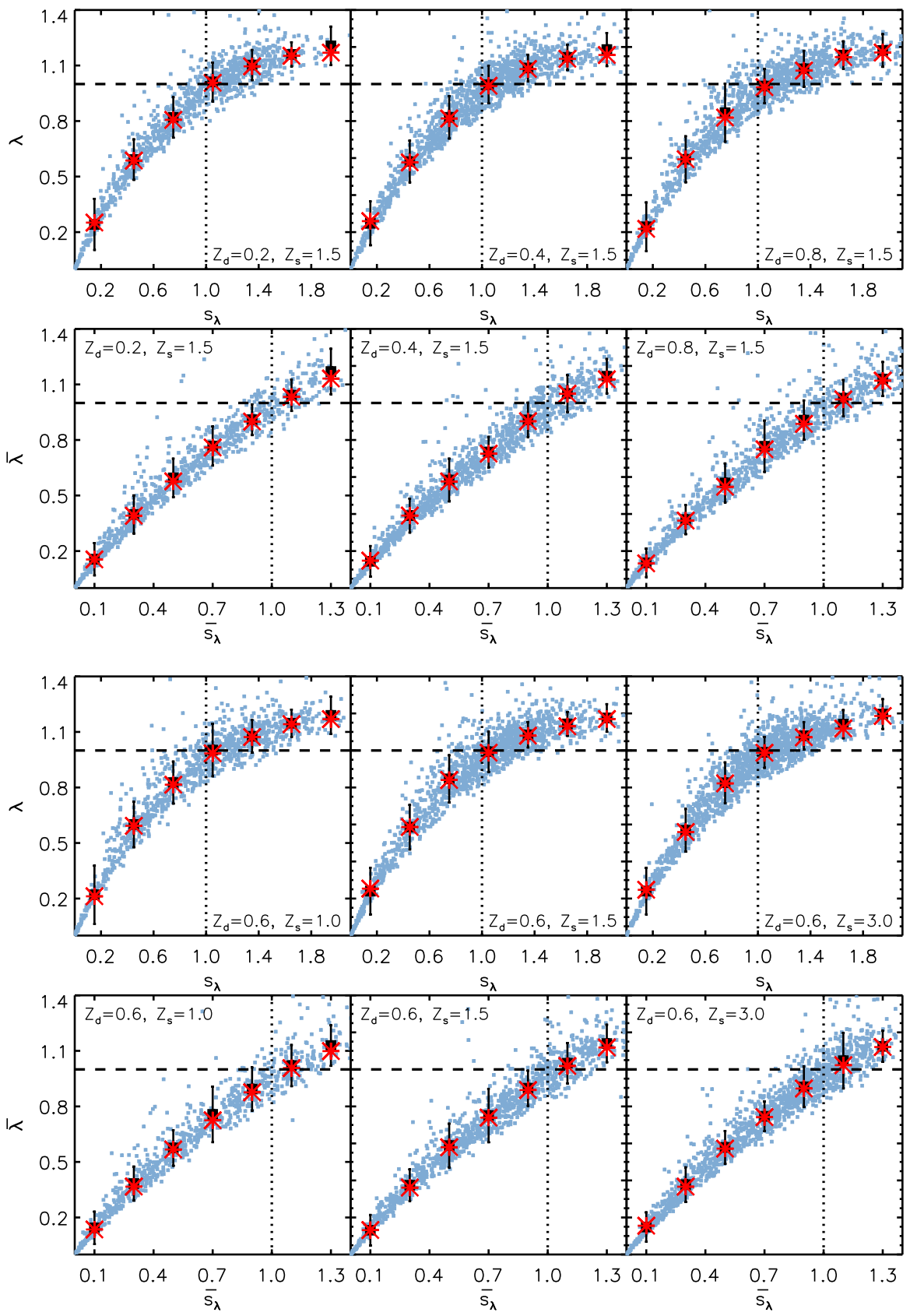

Figure C2. Distributions of $\lambda$ versus $s_{\lambda}$ and $\bar{\lambda}$ versus $\bar{s}_{\lambda}$ for all six samples with $z_{\mathrm{d}}$ and $z_{\mathrm{s}}$ indicated in the panels. On top of the scattered data (blue dots) the black squares (and red stars) with the error bars indicate the mean (and median) and its standard deviation within each bin. The dashed lines indicate where $\lambda=1$ and where $\bar{\lambda}=1$; while the dotted lines indicate where $s_{\lambda}=1$ and where $\bar{s}_{\lambda}=1$. 


\section{REFERENCES}

Agnello A., Auger M. W., Evans N. W., 2013, MNRAS, 429, L35

Auger M. W., Treu T., Bolton A. S., Gavazzi R., Koopmans L. V. E., Marshall P. J., Moustakas L. A., Burles S., 2010, ApJ, 724, 511

Barnabè M., Czoske O., Koopmans L. V. E., Treu T., Bolton A. S., 2011, MNRAS, 415, 2215

Barnabè M., Czoske O., Koopmans L. V. E., Treu T., Bolton A. S., Gavazzi R., 2009, MNRAS, 399, 21

Barnabè M., Koopmans L. V. E., 2007, ApJ, 666, 726

Bauer A. H., Baltay C., Ellman N., Jerke J., Rabinowitz D., Scalzo R., 2012, ApJ, 749, 56

Bolton A. S., Burles S., 2003, ApJ, 592, 17

Bolton A. S., Burles S., Koopmans L. V. E., Treu T., Gavazzi R., Moustakas L. A., Wayth R., Schlegel D. J., 2008, ApJ, 682, 964

Cardone V. F., Tortora C., Molinaro R., Salzano V., 2009, A\&A, 504, 769

Chornock R., Berger E., Rest A., Milisavljevic D., Lunnan R., Foley R. J., Soderberg A. M., Smartt S. J., Burgasser A. J., Challis P., Chomiuk L., Czekala I., Drout M., Fong W., Huber e. a., 2013, ApJ, 767, 162

Coe D., Moustakas L., 2009, ApJ, 706, 45

Dutton A. A., Treu T., 2014, MNRAS, 438, 3594

Falco E. E., Gorenstein M. V., Shapiro I. I., 1985, ApJ Letters, 289, L1

Finet F., Elyiv A., Surdej J., 2012, Memorie della Societa Astronomica Italiana, 83, 944

Gavazzi R., Treu T., Rhodes J. D., Koopmans L. V. E., Bolton A. S., Burles S., Massey R. J., Moustakas L. A., 2007, ApJ, 667, 176

Genel S., Vogelsberger M., Springel V., Sijacki D., Nelson D., Snyder G., Rodriguez-Gomez V., Torrey P., Hernquist L., 2014, MNRAS, 445, 175

Grillo C., Gobat R., Lombardi M., Rosati P., 2009, A\&A, 501, 461

Humphrey P. J., Buote D. A., Gastaldello F., Zappacosta L., Bullock J. S., Brighenti F., Mathews W. G., 2006, ApJ, 646,899

Jiang G., Kochanek C. S., 2007, ApJ, 671, 1568

Kolatt T. S., Bartelmann M., 1998, MNRAS, 296, 763

Koopmans L. V. E., Bolton A., Treu T., Czoske O., Auger M. W., Barnabè M., Vegetti S., Gavazzi R., Moustakas L. A., Burles S., 2009, ApJ Letters, 703, L51

Koopmans L. V. E., Treu T., Bolton A. S., Burles S., Moustakas L. A., 2006, ApJ, 649, 599

LSST Dark Energy Science Collaboration 2012, ArXiv eprints arXiv1211.0310

Napolitano N. R., Romanowsky A. J., Tortora C., 2010, MNRAS, 405, 2351

Nelson D., Pillepich A., Genel S., Vogelsberger M., Springel V., Torrey P., Rodriguez-Gomez V., Sijacki D., Snyder G. F., Griffen B., Marinacci F., Blecha L., Sales L., Xu D., Hernquist L., 2015, ArXiv e-prints

Oguri M., Kawano Y., 2003, MNRAS, 338, L25

Oguri M., Marshall P. J., 2010, MNRAS, 405, 2579

Quimby R. M., Oguri M., More A., More S., Moriya T. J., Werner M. C., Tanaka M., Folatelli G., Bersten M. C., Maeda K., Nomoto K., 2014, Science, 344, 396

Quimby R. M., Werner M. C., Oguri M., More S., More A.,
Tanaka M., Nomoto K., Moriya T. J., Folatelli G., Maeda K., Bersten M., 2013, ApJ Letters, 768, L20

Refsdal S., 1964, MNRAS, 128, 307

Rusin D., Kochanek C. S., 2005, ApJ, 623, 666

Rusin D., Kochanek C. S., Keeton C. R., 2003, ApJ, 595, 29

Schneider P., Sluse D., 2013, A\&A, 559, A37

Sérsic J. L., 1963, Boletin de la Asociacion Argentina de Astronomia La Plata Argentina, 6, 41

Sonnenfeld A., Treu T., Gavazzi R., Suyu S. H., Marshall P. J., Auger M. W., Nipoti C., 2013, ApJ, 777, 98

Suyu S. H., Auger M. W., Hilbert S., Marshall P. J., Tewes M., Treu T., Fassnacht C. D., Koopmans L. V. E., Sluse D., Blandford R. D., Courbin F., Meylan G., 2013, ApJ, 766, 70

Suyu S. H., Marshall P. J., Auger M. W., Hilbert S., Blandford R. D., Koopmans L. V. E., Fassnacht C. D., Treu T., 2010, ApJ, 711, 201

Suyu S. H., Treu T., Hilbert S., Sonnenfeld A., Auger M. W., Blandford R. D., Collett T., Courbin F., Fassnacht C. D., Koopmans L. V. E., Marshall P. J., Meylan G., Spiniello C., Tewes M., 2014, ApJ Letters, 788, L35

Tortora C., Napolitano N. R., Romanowsky A. J., Capaccioli M., Covone G., 2009, MNRAS, 396, 1132

Treu T., Koopmans L. V. E., 2002, MNRAS, 337, L6

Treu T., Koopmans L. V. E., 2004, ApJ, 611, 739

van de Ven G., Mandelbaum R., Keeton C. R., 2009, MNRAS, 398, 607

Vogelsberger M., Genel S., Sijacki D., Torrey P., Springel V., Hernquist L., 2013, MNRAS, 436, 3031

Vogelsberger M., Genel S., Sijacki D., Torrey P., Springel V., Hernquist L., 2014, MNRAS, 438, 3607

Vogelsberger M., Genel S., Springel V., Torrey P., Sijacki D., Xu D., Snyder G., Bird S., Nelson D., Hernquist L., 2014, Nature, 509, 177

Vogelsberger M., Genel S., Springel V., Torrey P., Sijacki D., Xu D., Snyder G., Nelson D., Hernquist L., 2014, MNRAS, 444, 1518

Walsh D., Carswell R. F., Weymann R. J., 1979, Nature, 279,381 